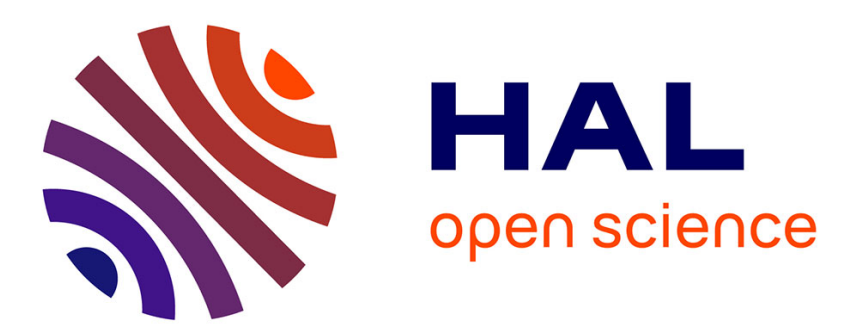

\title{
Wave distribution functions estimation of VLF electromagnetic waves observed onboard Geos 1
}

François Lefeuvre, Michel Parrot, C. Delannoy

\section{To cite this version:}

François Lefeuvre, Michel Parrot, C. Delannoy. Wave distribution functions estimation of VLF electromagnetic waves observed onboard Geos 1. Journal of Geophysical Research, 1981, 86 (A4), pp.2359. 10.1029/JA086iA04p02359 . insu-03206544

\section{HAL Id: insu-03206544 https://hal-insu.archives-ouvertes.fr/insu-03206544}

Submitted on 23 Apr 2021

HAL is a multi-disciplinary open access archive for the deposit and dissemination of scientific research documents, whether they are published or not. The documents may come from teaching and research institutions in France or abroad, or from public or private research centers.
L'archive ouverte pluridisciplinaire HAL, est destinée au dépôt et à la diffusion de documents scientifiques de niveau recherche, publiés ou non, émanant des établissements d'enseignement et de recherche français ou étrangers, des laboratoires publics ou privés. 
JOURNAL OF GEOPHYSICAL RESEARCH, VOL. 86, NO. A4, PAGES 2359-2375, APRIL 1, 1981

WAVE DISTRIBUTION FUNCTIONS ESTIMATION OF VLF ELECTROMAGNETIC WAVES

OBSERVED ONBOARD GEOS 1

F. Lefeuvre, M. Parrot, and C. Delannoy

Centre de Recherches en Physique de l'Environnement, 45045 Orléans Cédex, France

\begin{abstract}
Two methods to determine the electromagnetic wave distribution function are presented. The first is based on the use of the dirichlet kernels and provides us with a local average. It has the disadvantage, however, of a nonsystematic approach to positive solutions. The second uses the maximum entropy concept. It leads to particular solutions that are smooth and positive everywhere. The two methods are shown to be complementary. Applications to VLF electromagnetic waves observed onboard Geos 1 are discussed. One of the most striking results is that the wave energy of the natural VLF emissions is generally concentrated within two wave packets whose wave normals are approximately in the same off-meridian plane and oriented in the same way relative to the direction of the earth's magnetic field. It is suggested that those two wave packets have a common source.
\end{abstract}

\section{Introduction}

Electromagnetic wave fields are generally analyzed in terms of the wave normal direction [Grard, 1968; Shawhan, 1970; McPherron et a1., 1972; Means, 1972; Arthur et al., 1976; Cornilleau-Wehrlin et al., 1976; Kodera et al., 1977; Loisier et al., 1979]. A more realistic description in terms of wave distribution function has been proposed for some time [Storey, 1971; Storey and Lefeuvre, 1974, 1979] but has only been applied up to now on a very limited set of data [Lefeuvre, 1977; Lefeuvre and Delannoy, 1979]. Now a certain number of wave distribution functions have been determined from the Geos 1 multi-component data; it is time to give a first appraisal of what can be expected from this type of analysis.

When the field can be regarded as that of a single plane wave, things are clear. At a given frequency $\omega$ and for a given mode (ordinary or extraordinary), one can always determine a direction of propagation from the measurements of the six electromagnetic components of the field ( 3 electric and 3 magnetic) or even from the measurements of only five components [Grard, 1968; Shawhan, 1970]. When the only available (or reliable) measurements are of the magnetic components, the same information can be obtained, but with an ambiguity in sign. One cannot ascertain whether the wave propagates in the direction of the earth's magnetic field $\vec{B}_{o}$ or in the opposite direction. Except for this ambiguity the solution to the problem is unique [Lefeuvre, 1977]. The existing uncertainty is due only to errors made during the recording and handiing of the data.

The situation in which the field is that of

Copyright 1981 by the American Geophysical Union. a single plane wave is probably not common. Natural noise fields are more likely to be composed of a continuum of superimposed plane waves of different frequencies and propagating in different directions without any mutual phase coherence [Storey, 1971]. The properties of such an incoherent and randon wave field can only be described statistically. Storey and Lefeuvre [1974, 1979] have proposed to characterize it by means of a function called the wave distribution function (WDF), which specifies how the wave energy density is distributed with respect to the angular frequency (s) and to the direction of propagation $\vec{k}(\vec{k}=\vec{k} /|\vec{k}|$, with $\vec{k}$ the wave normal direction). The difference is that the solution to such a problem is not unique. At a given frequency, for a known mode of propagation, there are infinities of WDFs that can explain the statistics of the wave field components. The uncertainty exists even if there are no processing errors in the data.

In discarding the plane wave hypothesis we are compelled to seek a function knowing it will never be the 'true' one. This is a common dilemma in physics. However, we have made use of the considerable findings by earth physicists Backus and Gilbert [1968, 1970], Wiggins [1972], and Jackson [1972], etc.

There are some constraints that make the solution to our problem far easier than it appears. We consider those of positivity and stability only (this choice does not rule out other possibilities). One cannot readily accept an energy function with negative values. Although mathematically less obvious, the stability constraint is also physically easy to understand. In dealing with 'noisy' data we do not want to have the solutions altered by a slight variation. We cannot measure the power of these two constraints, but have observed that whatever the inversion methods, the solutions that respect them are very similar. This lends credibility to our efforts.

In this paper we shall only deal with two methods. The first is a linear method based on the dirichlet kernels [Backus and Gilbert, 1968]. It leads to a solution that does not always obey the positivity constraint, but results in an averaged solution. The second is based on maximum entropy concepts [Lefeuvre, 1977; Lefeuvre and Delannoy, 1979]. It leads to a solution that is smooth and positive everywhere. In a continuation [Buchalet and Lefeuvre, this issue], a third method will be introduced. It consists of identifying models with one or more plane waves.

Magnetic data analysis is strongly emphasized in this paper because measurements of the magnetic components are more feasible than those of the electric components. However, even if the electric measurements are not sufficiently accu- 
rate to improve the resolution of the solutions, they have often been found consistent enough to resolve the $180^{\circ}$ ambiguity in propagation direction noted above.

In section 2 we present the two methods of WDF defined above. In section 3 the quality of the Geos 1 data is discussed. Examples of applications are given in section 4. Finally, section 5 offers some provisional conclusions.

Several assumptions and approximations are made in order to simplify the problem. We consider only the whistler mode of propagation, and all statistical properties of the wave fields are taken to be stationary in time and space. The plasma is cold and collisionless, characterized by the electron plasma frequency $\Pi_{e}$ and the electron gyrofrequency $\Omega_{e}$ and assumed to be uniform on a scale much larger than a wavelength. The point of observation is fixed with respect to the plasma.

\section{Methods}

We adopt Cartesian coordinates system Oxyz, where the $O z$ axis is parallel to the earth's magnetic field $\overrightarrow{\mathrm{B}}_{\mathrm{O}}$, $0 \mathrm{x}$ is in the meridian containing the point of observation, while oy completes the orthogonal set and is oriented eastward. In this system the axial components of the electric and magnetic fields of the wave at the point of observation are respectively denoted $E_{x}, E_{y}, E_{z}$ and $H_{x}, H_{y}$, and $H_{z}$. From these varia$b$ les a general electric vector $\varepsilon$, with six components, is defined as follows

$$
\epsilon_{1,2,3}=E_{x, y, z} ; \varepsilon_{4,5,6}=z_{0} H_{x, y, z}
$$

where $\mathrm{Z}_{o}$ is the wave impedance of free space. Let $\varepsilon_{i}$ be any component of this vector $(i=1, \ldots 6)$. Then, at a given frequency $w$, the spectral matrix $s_{i j}(\omega)$ is defined in a way that each of its 36 elements $s_{i j}(\omega)$ is either the mean auto power spectrum of the field component $E_{i}$ (if $i=j$ ), or the mean cross-power spectrum of the components $\varepsilon_{i}$ and $E_{j}$ (if $i$ is not equal to $j$ ).

Storey and Lefeuvre $[1974,1979]$ have shown that for a given mode of propagation (here, the whistler mode), at the frequency $\omega$ the WDF is related to the elements of the spectral matrix by the following equation:

$s_{i j}(\omega)=\frac{\pi}{2} \oiint a_{i j}(\omega, \cos \theta, \phi) F(\omega, \cos \theta, \phi) d \sigma$

Here, $\theta$ is the angle between the direction of propagation $\vec{k}$, and the earth's magnetic field $\vec{B}_{0}$, and $\phi$ the azimuthal angle whose origin is in the ox, oz plane. $F(\omega, \cos \theta, \phi)$ is the WDF. The kernels $a_{i j}(\omega, \cos \theta, \phi)$ implicitly depend on the plasma parameters. Their analytical expressions are given in Storey and Lefeuvre [1980]. $\mathrm{d} \sigma=\mathrm{d} \cos \theta \mathrm{d} \phi$ is the surface element. The integral is taken over the surface of the sphere of unit radius.

Note that in all the mathematical treatments, $\theta$ is supposed to vary between 0 and $\pi$ (or $\cos \theta$ between 1 and -1 ) and $\phi$ between 0 and $2 \pi$. But in the interpretation of the results in sections 4 and 5 , when dealing with propagation in planes $\phi=c^{s t e}$, we will find it more convenient to have $\theta$ vary between $-\pi$ and $+\pi$ and $\phi$ between 0 and $\pi$. When the reader sees a negative value for $\theta$, he will immediately understand that the latter convention has been chosen.

For the sake of simplicity, at the frequency $w$, the set of integral equations $(2-a)$ can be rewritten.

$$
\mathrm{P}_{\mathbf{k}}=\frac{\pi}{2} \oiint \mathrm{q}_{\mathbf{k}}(\cos \theta, \phi) \mathrm{F}(\cos \theta, \phi) \mathrm{d} \sigma
$$

$P_{k}$ and $q_{k}$ are associated with the real and imaginary parts of the spectra $S_{i j}$ and of the kernels $a_{i j}\left(P_{1}=S_{11}, P_{2}=\operatorname{Re}\left(S_{12}\right), P_{3}=\operatorname{Im}\left(S_{12}\right)\right.$, $\ldots P_{36}=S_{66} ; q_{1}=a_{11}, q_{2}=\operatorname{Re}\left(a_{12}\right), \ldots$. $\left.q_{36}=a_{66}\right)$. The estimated values of the data

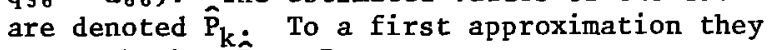
are such that $\hat{\mathrm{P}}_{\mathrm{k}}=\mathrm{P}_{\mathrm{k}}$.

We tackle the following problem : given data from which we can estimate the 36 elements of the spectral matrix, to what extent can the WDF be determined and how should this be done?

Several approaches have been considered in previous papers [Lefeuvre and Storey, 1977; Lefeuvre, 1977; Lefeuvre and Delannoy, 1979]. Here we shall deal with the two methods based on the dirichlet kernels and the maximum entropy concepts. Although the first method does not lead to strictly positive solutions, we stress its importance in clarifying a number of points, mainly the stability constraint, and in helping us understand the second method.

Dirichlet kernels method. The dirichlet kernels method is a particular case of the general method of Backus and Gilbert [1968]. This method consists of constructing at each point $\left(\cos \theta_{0}, \phi_{0}\right)$ a local average $\langle F(\cos \theta, \phi)\rangle$ $\cos \theta_{o}, \phi_{o}$ of any possible model $\mathrm{F}(\cos \theta, \phi)$. It can be written

$$
\begin{aligned}
& <\mathrm{F}(\cos \theta, \phi)\rangle_{\cos \theta_{0}, \phi_{0}}= \\
& \oiint_{\mathrm{F}(\cos \theta, \phi) \mathrm{A}\left(\cos \theta, \phi ; \cos \theta_{0}, \phi_{0}\right) \mathrm{d} \sigma}
\end{aligned}
$$

where the function $\mathrm{A}\left(\cos \theta, \phi ; \cos \theta_{0}, \phi_{0}\right)$ is an averaging kernel that is, at each point $\left(\cos \theta_{0}\right.$, $\left.\phi_{0}\right)$, a linear combination of the $q_{i}(\cos \theta, \phi)$

$$
\mathrm{A}\left(\cos \theta, \phi ; \cos \theta_{\mathrm{o}}, \phi_{\mathrm{o}}\right)=\sum_{\mathrm{k}=1}^{36} \alpha_{\mathrm{k}}^{\mathrm{o}} \mathrm{q}_{\mathrm{k}}(\cos \theta, \phi)
$$

The unknown parameters $\alpha_{k}^{o}$ are estimated at each point $\left(\cos \theta_{0}, \phi_{0}\right)$. They are chosen in such a way that A becomes unimodular ( $\iint \mathrm{Adcos} \theta \mathrm{d} \phi=1$ ) and 'looks like' a dirac distribution $\delta(\cos \theta-$ $\left.\cos \theta_{0}, \phi-\phi_{0}\right)$. Then it is found first of a11 that the local average ( 3 ) is the same for all the possible models $\mathrm{F}(\cos \theta, \phi)$, and second, that it can be expressed as a linear combination of the data

$$
\langle F(\cos \theta, \phi)\rangle_{\cos \theta_{o}, \phi_{0}}=\sum_{k=1}^{36} \alpha_{k}^{\circ} \hat{P}_{k}
$$

The width of the peak in $A\left(\cos \theta, \phi ; \cos \theta_{0}, \phi_{0}\right)$ measures the resolving power of the data near $\left(\cos \theta_{0}, \phi_{0}\right)$.

Obviously, the solution depends on the ' $\delta$-ness' criterion that has been chosen. It generally does not exactly satisfy the data $\hat{P}_{k}$ and cannot be considered to be an exact solution [Backus and Gilbert, 1968]. 
The dirichlet kernels approach, proposed also by Backus and Gilbert, differs slightly from the foregoing in the sense that the solution is an a priori one assumed to be a linear combination of the kernels. But it appeals to the same concept since it can also be interpreted as a local average. By definition, it is an exact solution. Suitable $\delta$-ness criteria may eventually lead to smoother solutions that better obey the positivity constraint. However, we prefer the dirichlet kernels solution because it is analytical (which means that its parameters are known functions of $\cos \theta$ and $\phi$ ) and thus requires less computation.

To derive the solution, we first define, in the space of the $q_{k}$ 's, a set of orthogonal functions analagous to the dirichlet kernels. If the $\mathrm{N} \quad \mathrm{q}_{\mathrm{k}}$ 's are linearly independent, which we presently assume, they generate an $\mathrm{N}$ dimensional space $V$. Let $\Pi_{1}, \ldots . \Pi_{N}$ be any orthonormal basis for $V$. Then the $q_{k}$ 's are a linear combination of the $\Pi_{k}$ 's and vice versa. They are related by the equation

$$
\pi_{k}=\sum_{L=1}^{N} T_{k L} q_{L}
$$

where the orthogonal matrix $\mathrm{T}$ can be chosen in such a way that [Lefeuvre and Delannoy, 1979]

$$
\mathrm{T}_{\mathrm{kL}}=\mathrm{V}_{\mathrm{Lk}} / \lambda_{\mathrm{k}}^{1 / 2}
$$

the $\lambda_{k}$ being the eigenvalues, ranked in decreasing order, of the matrix $B$ defined by

$$
B_{k L}=\oiint q_{k}(\cos \theta, \phi) q_{L}(\cos \theta, \phi) d \sigma
$$

and the $\mathrm{V}_{\mathrm{L} k \mathrm{k}}(\mathrm{L}=1, \ldots \mathrm{N})$, the associated eigenvectors.

In the new basis, the data $\hat{\mathrm{P}}_{k}$ are transformed into the data $\hat{\mathrm{P}}_{\mathrm{k}}$ '

$$
\hat{\mathrm{P}}_{\mathrm{k}}^{\prime}=\sum_{\mathrm{L}=1}^{\mathrm{N}} \mathrm{T}_{\mathrm{kL}} \hat{\mathrm{P}}_{\mathrm{L}}
$$

and it is easily seen that the function

$$
F(\cos \theta, \phi)=\sum_{k=1}^{N} P_{k}^{\prime} \Pi_{k}(\cos \theta, \phi)
$$

satisfies all the transformed data $\hat{\mathrm{P}}_{\mathbf{k}}{ }^{\prime}$. This solution can be interpreted as a local average of all the possible solutions near any point $\left(\cos \theta_{0}, \phi_{0}\right)$ of the space $\cos \theta, \phi$. Then the averaging kernel $A$ can be expressed [Sabatier, 1974] as

$$
\begin{aligned}
& \mathrm{A}\left(\cos \theta, \phi ; \cos \theta_{0}, \phi_{\mathrm{o}}\right)= \\
& \quad \sum_{\mathrm{k}=1}^{\mathrm{N}} \mathrm{P}_{\mathrm{k}} \Pi_{\mathrm{k}}(\cos \theta, \phi) \Pi_{\mathrm{k}}\left(\cos \theta_{\mathrm{o}}, \phi_{\mathrm{o}}\right)
\end{aligned}
$$

Now, are the $q_{k}$ 's obtained for given values of $\omega, \Pi_{e}$, and $\Omega_{e}$ linearly independent? The best way to answer this question is probably to compute the eigenvalues of the matrix $B$ defined in (8). If one or more eigenvalues are equal to zero, a corresponding number of kernels are defined as linearly dependent. If one or more eigenvalues are small relative to the maximum ei- genvalue, a corresponding number are almost 1inearly dependent.

In order to illustrate this point we consider the $9 \times 9$ matrix B of magnetic components only. Assuming that its eigenvalues are ranked in decreasing order so that $\lambda_{1}$ is the maximum eigenvalue while $\lambda_{9}$ is the minimum one, we have represented in Figure 1 the variations of the ratios $\lambda_{i} / \lambda_{1}(i=2 \ldots 9)$ versus $\omega / \Omega_{e}$. The plasma frequency $\Pi_{e}$ does not sensibly affect these ratios in the whistler mode [Storey and Lefeuvre, 1980] and has been given an arbitrary value $\left(\Pi_{e}=50 \mathrm{kHz}\right)$. For $\omega / \Omega_{e}$ close to zero (the low part of the Geos 1 orbit), there is one small eigenvalue, and 8 kernels $q_{1}, \ldots$ $\mathrm{q}_{\mathrm{N}}$ can be considered as being practically $1 \mathrm{i}-$ nearly independent. For $\omega / \Omega_{e}$ close to 0.5 (the apogee of the Geos 1 orbit), one eigenvalue is essentially equal to zero and two others are small relative to the maximum eigenvalue. It is not possible to say that there are more than 6 linearly independent values.

The situation is more confused when the electric antennas are considered. Having previously identified three linear dependencies [Lefeuvre, 1977; Storey and Lefeuvre, 1980], we know that there is always a minimum of three null eigenvalues. The number of very small eigenvalues varies with the ratio $\omega / \Omega_{e}$ and the $\Pi_{e} / \Omega_{e}$. It is generally between 18 and 23 .

The derivation of the dirichlet kernels solution assumes (see (7)) no null eigenvalues. If any exist, the associated eigenvectors must be removed. Then the orthogonalization matrix becomes a $M_{o} \times N$ matrix, $M_{0}$ being the number of non-null eigenvalues. As a consequence we have only to consider $M_{o}$ transformed kernels $\Pi_{k}$ and data $\hat{\mathrm{P}}_{\mathrm{k}}$. O Obviously there is no loss of information in doing so since we are only eliminating redundant kernels and data.

What about the items of information associated with the small but non-null eigenvalues? Their effect on the solution is very easy to calculate [Gilbert, 1971]. When the data are slightly varied, the variation of $F(\cos \theta, \phi)$ can be expressed

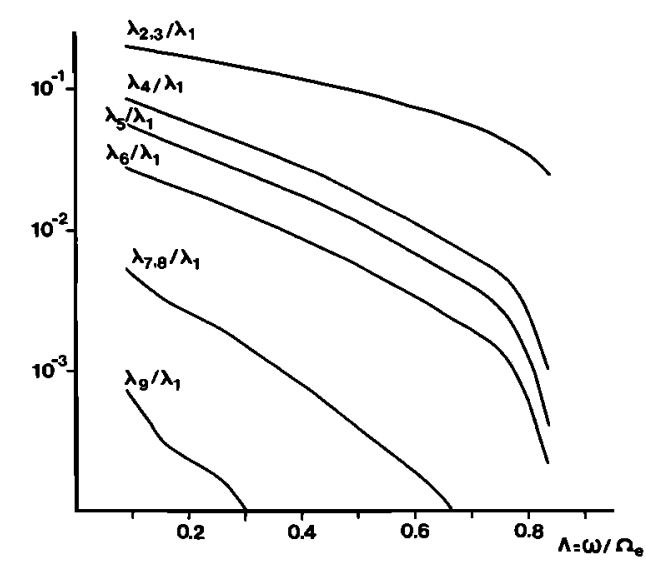

Fig. 1. Linear dependence of the kernels $a_{i j}$ versus $\omega / \Omega_{e}$, with $\omega$ the wave angular frequency and $\Omega_{\mathrm{e}}$ the electron angular gyrofrequency. The $\lambda_{i}$ 's are the eigenvalues of the matrix $B$ defined in (8); they are ranked in decreasing order (see text). 
$\left\langle\delta F^{2}\right\rangle=\sum_{k=1}^{M_{O}} \sum_{L=1}^{M_{O}} \frac{\pi_{k} \Pi_{L}}{\sqrt{\lambda_{k} \lambda_{L}}} \sum_{i=1}^{M_{0}} \sum_{j=1}^{M_{O}} v_{i k} v_{j L}\left\langle\delta \hat{P}_{i} \delta \hat{P}_{j}\right\rangle$

As a first approximation, considering all the elements $\left\langle\delta \hat{\mathrm{P}}_{i} \delta \hat{\mathrm{P}}_{j}\right\rangle$ to be of the same order, we see that $\left\langle\delta \mathrm{F}^{2}\right\rangle$ is proportional to $\lambda^{-1}$. The items of information associated with the sma1lest eigenvalues, which by definition are the poorest, make the solutions completely unstable. They must obviously be removed in the same way as the items of information associated with the null eigenvalues. The number of items that must be considered is not $M_{O}$ but a number $M \leqslant M_{O}$, which must also be defined.

Theoretically, one should not only define the number $M$ but also indicate in particular the $N-M$ items of information that must be removed. However, we do not commit a large error in assuming that the linear dependency is exactly proportional to $\lambda^{-1}$; that is, the items of information associated with the smallest eigenvalue are the most linearly dependent [Lefeuvre, 1977]. This simplification makes the truncation much easier. It also has the great advantage of not depending on the quality of the estimates of errors in the data, which is generally quite poor. A review of more sophisticated truncation methods can be found in Sabatier [1976].

The truncation has two effects. First, it reduces the ability of the solution to fit all the original data $\widehat{\mathrm{P}}_{i}$; the first $M$ transformed data $\hat{\mathrm{P}}_{\mathrm{k}}$ are exactly satisfied by the solution, but according to ( 9 ) the last of the $\mathrm{N}-\mathrm{M}$ data are supposed to be equal to zero, which is not true. Second, it reduces the resolving power of the method; as shown in Figure 2, uncertainty in the localization of the peak and the width of the averaging kernel $\mathrm{A}\left(\cos \theta, \phi ; \cos \theta_{0}, \phi_{0}\right)$ increases when $M$ decreases. A compromise must be found in order to derive a solution which is stable ( $M$ as low as possible), but which also fits the original $\hat{\mathrm{P}}_{\mathbf{k}}$ and has a sufficient resolving power ( $M$ as large as possible).

As we shall see later, the choice of the $M$ values is very restricted. For $N=9$ for instance, according to the values of the ratios $\omega / \Omega_{e}$ and $\Pi_{e} / \Omega_{e}$, we can only take $M=7$ or $M=8$, or just $M=7$. Then it is not unreasonable to forecast an a posteriori test of the validity of the truncation, which we have done. Such a test is based on the estimation of two quality parameters: the stability parameter $Q$ and the prediction parameter $P_{r}$ [Lefeuvre, 1977; Lefeuvre and Delannoy, 1979].

The stability parameter has been defined as being the ratio between the mean-square error of the solution and the mean-square value of the solution itself. It is written

$$
Q=\frac{\oiint\left\langle\delta F^{2}(\cos \theta, \phi)\right\rangle d \sigma}{\oiint F^{2}(\cos \theta, \phi) d \sigma}
$$

Substituting (11) into (10), one obtains

$$
Q=\frac{\sum_{k=1}^{M}\left\langle\delta \hat{\mathrm{P}}_{\mathrm{k}}{ }^{\prime 2}\right\rangle}{\sum_{\mathrm{k}=1}^{\mathrm{M}} \hat{\mathrm{P}}_{\mathrm{k}}{ }^{\prime 2}}
$$

A solution is considered stable if $Q \leqslant 1$. The reliability of the exact value of $Q$ is obviously a function of the accuracy of the estimation of errors in the data.

The quality of the fit to the original data is measured by the parameter $P_{r}$

$$
P_{r}=\frac{1}{N} \sum_{k=1}^{N} \frac{\left(P_{k}-\hat{P}_{k}{ }^{r}\right)^{2}}{\left\langle\delta \hat{P}_{k}{ }^{2}\right\rangle}
$$

where the ${P_{k}}^{r}$ are the data reconstructed from the solutions. The term 'prediction parameter' we use for $P_{r}$ may seem ambiguous, since (15) is a measure of the residuals in the space of the original data $\hat{\mathrm{P}}_{\mathrm{k}}$. However, we keep it to point out that it is also an estimation of the truncation validity. Subject to the same restrictions as for $Q$, we are satisfied when $P_{r}$ is of the same order as the errors in the data, i.e., when $P_{r} \simeq 1$.

Both the stability parameter and the prediction parameter will be used to qualify the maximum entropy solution we examine now.

The maximum entropy method. The maximum entropy solution has been discussed in a previous paper [Lefeuvre and Delannoy, 1979]. It has the advantage of being smooth and respects the positivity constraint on $\mathrm{F}(\cos \theta, \phi)$. We only recall here its main characteristics.

The entropy of the function $F(\cos \theta, \phi)$ can be expressed as

$$
\oiint F(\cos \theta, \phi) \log F(\cos \theta, \phi) d \sigma
$$

Its maximization subject to the constraints (2-a) produces the solution

$\mathrm{F}(\cos \theta, \phi)=\exp \left\{-1+\sum_{\mathrm{k}=1}^{\mathrm{N}} \mu_{\mathrm{k}} \mathrm{a}_{\mathrm{k}}(\cos \theta, \phi)\right\}$

The parameters $\mu_{k}$ are Lagrange multipliers. They are chosen to satisfy the inequality

$$
t_{\delta \vec{P}} W \delta \overrightarrow{\mathbf{P}} \leqslant \varepsilon
$$

where $\delta \vec{P}$ is the error vector of which the $k^{\text {th }}$ component is $\delta \mathrm{P}_{k}=\hat{\mathrm{P}}_{\mathrm{k}}-\mathrm{P}_{\mathbf{k}} \mathrm{r}$, and $\mathrm{t}_{\delta} \overrightarrow{\mathrm{P}}$ is the transposed vector, $W$ is the matrix of the measurement errors in $\hat{\mathrm{P}}_{k}$, given by $\mathrm{W}_{\mathrm{kL}}=\left\langle\delta \mathrm{P}_{\mathrm{k}} \delta \mathrm{P}_{\mathrm{L}}\right\rangle$ and $\varepsilon$ is a fixed, known, positive number that denotes the precision with which we intend to fit the data.

The linear dependency of the kernels $q_{1}$, .... $q_{N}$ causes the same difficulties as in the previous method. The Lagrange multipliers $\mu_{k}$ are linearly dependent, and if a solution exists, it is essentially unstable [Lefeuvre and Delannoy, 1979]. To obtain a stable solution, we are led to remove a certain amount of information. The procedure we propose is the same as in the dirichlet kernels method. We consider the first $M$ transformed kernels $\Pi_{k}$ and data $\widehat{\mathrm{P}}_{k}$ ', the number $M$ possibly being different from the one found suitable for the dirichlet kernels method. Then we seek the new maximum entropy solution, which has the expression 
$\Theta$

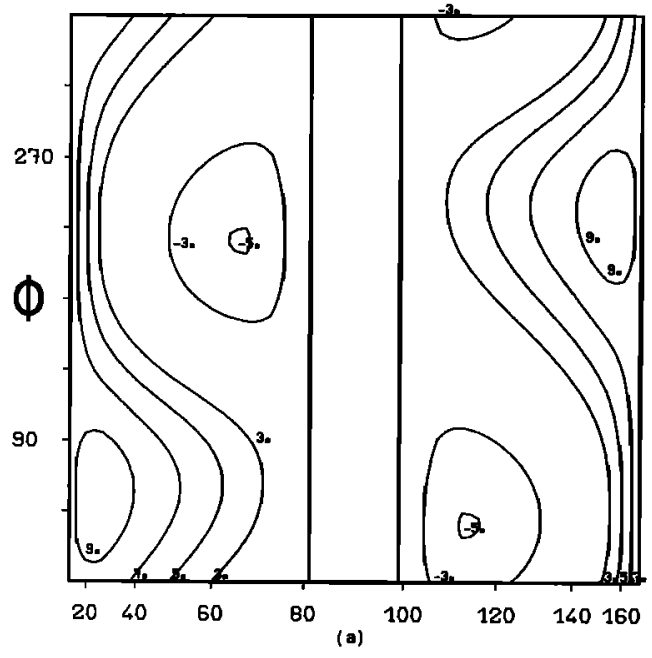

$\Theta$

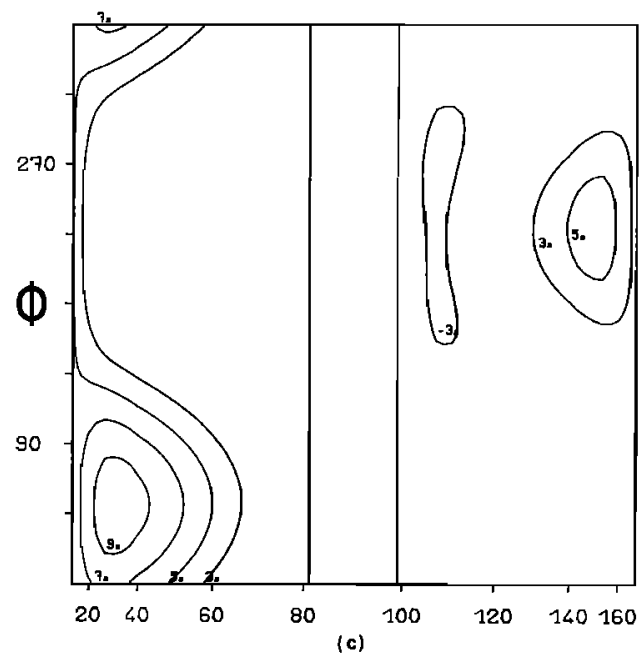

$\Theta$

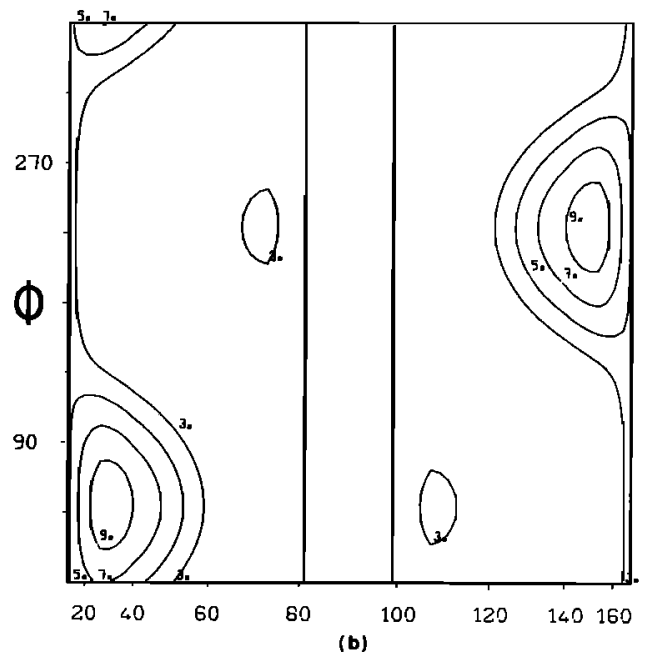

$\Theta$

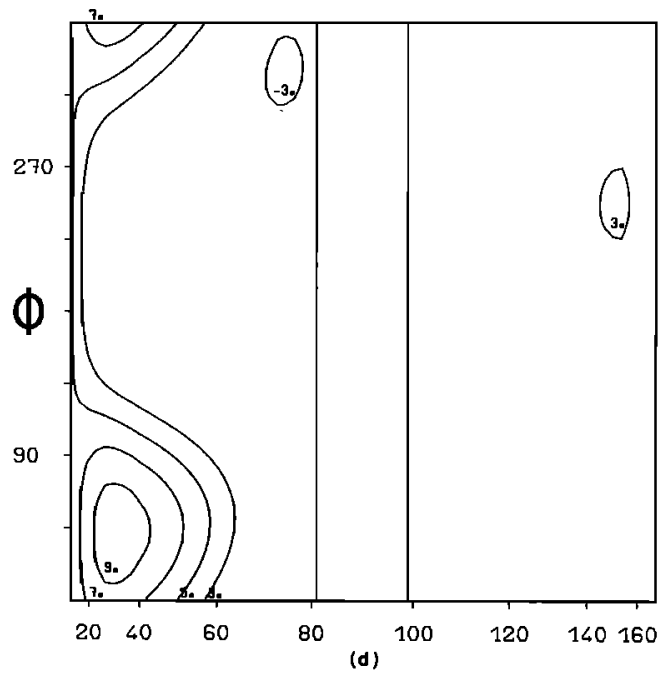

Fig. 2. Contours of the averaging kernel $A\left(\cos \theta, \phi ; \cos \theta_{0}, \phi_{0}\right)$ for $\theta_{0}=30^{\circ}$ and $\phi_{0}=30^{\circ}$. A has been normalized to 10 . The scale in amplitude is linear. There is a nonpropagating zone around $\theta=\pi / 2$ and it is bounded by $|\cos \theta|=\omega / \Omega_{e}$ with $\omega$ and $\Omega_{e}$ the wave angular frequency and the electron-angular gyrofrequency. Here $\omega=$ $0.625 \mathrm{kHz}, \Omega_{e}=4 . \mathrm{kHz}$. The electron plasma frequency $\Pi_{e}=18$. kHz. We first consider the magnetic kernels only $(\mathrm{N}=9)$, then the magnetic and the electric $(\mathrm{N}=36)$; $M$ is the number of transformed data we consider. (a) $N=9, M=5$. (b) $N=9, M=8$. (c) $\mathrm{N}=36, \mathrm{M}=12$. (d) $\mathrm{N}=36, \mathrm{M}=16$.

$\mathrm{F}(\cos \theta, \phi)=\exp \left[-1+\sum_{k=1}^{M} \mu_{k} \Pi_{k}(\cos \theta, \phi)\right\}$

The new Lagrange multipliers must satisfy the inequality

$$
{ }^{t} \delta \vec{P}^{\prime} W^{\prime} \delta \vec{P}^{\prime} \leqslant \varepsilon^{\prime}
$$

where $\delta \overrightarrow{\mathrm{P}}^{\prime}$ is the transformed error vector, $W^{\prime}$ is the matrix of the measurement errors in $\widehat{\mathrm{P}}_{\mathrm{k}}$, given by $W^{\prime}{ }_{k L}=\left\langle\hat{\delta} \mathrm{P}_{\mathrm{k}}{ }^{\prime} \hat{\delta} \mathrm{P}_{\mathrm{L}}{ }^{\prime}\right\rangle$, and $E^{\prime}$ the precision with which we intend to fit the $M$ data $\hat{P}_{k}$. We practically admit a model error of the same or- der as the errors in the data. Therefore $\varepsilon^{\prime}$ is set to $M$.

The solution of (20) requires that we supply initial values for the parameters $\mu_{k}$. Such initial values can be deduced from a dirichlet kernels solution [Lefeuvre and Delannoy, 1979]. However, we have observed a rapid convergence when starting from a uniform solution, i.e., starting will all the $\mu_{k}$ equal to zero.

Although the propagation of the instability is not exactly the same as for the dirichlet kernels solution, the stability and prediction parameters, respectively defined in (13) and (15), have been found to be good quality indicators for the maximum entropy solution [Lefeu- 
vre and Delannoy, 1979]. Obviously, one must be very careful in interpreting them. They generally are much more sensitive to an increase in $M$, and we can slightly pass the threshold $Q=1$ without noting any instability in the solution. This point will be more fully discussed in section 4 .

\section{Data}

The field data are signals obtained by continuous measurements of the electric and magnetic components of the field. The electric com ponents are measured by the short axial electric sensors. In the frame of reference of the satellite they are noted $e_{x}, e_{y}, e_{z}$. However, the y component can also be obtained from the long radial antennas. In that case it is noted as $\mathrm{E}_{\mathrm{Y}}$. The magnetic components are measured at the lowest frequencies $(<450 \mathrm{~Hz})$ by the ULF magnetic sensors and at other frequencies by the VLF magnetic sensors. To distinguish between them they are respectively labeled $B_{x}, B_{y}, B_{z}$ and $b_{x}, b_{y}$, and $b_{z}$. For further details see S-300 Experimenters [1979].

Because of the incorrect deployment of one of the axial booms, the electric components measured onboard Geos 1 are not in an orthogonal system. However, as long as the three electric components are given, there is no difficulty in recalculating them according to the appropriate orthogonal system. This correction is systematically done here. The only remaining uncertainty is due to the imperfect estimation of the length of the incompletely deployed boom. This uncertainty is negligible relative to the ones we shall encounter later.

Before being transmitted to the ground, the field data are subjected to several onboard treatments, as discussed below.

Six swept frequency analyzers (SFA) process the six components. These analyzers can select a $300-\mathrm{Hz}$ bandwidth in the range $150 \mathrm{~Hz}-77 \mathrm{kHz}$, in steps of $300-\mathrm{Hz}$. The six signals are simultaneously transposed to the low-frequency range and filtered in the $150-$ to $450-\mathrm{Hz}$ band. The transposition dephases equally the six signals, so the relative phases stay unchanged. Knowing the transfer function (phase and amplitude) of the filters, one can reconstruct the original wave forms.

Before telemetry, the waveforms are sampled at a rate of 1488 samples per second, which is slightly above the Shannon period. An important drawback of this sampling is that the operations are not simultaneous on the six channels. This time-shift causes a nonnegligible phase-shift, for which a correction must be made.

Between the sensor inputs and the telemetry outputs, there are many opportunities for distortions (sensors, preamplifiers, analyzers, switches, etc.). They globally act as a filter. The transfer function of this equivalent filter is estimated from calibrations made onboard the satellite.

The measurements of electric components are subject to other uncertainties (P.M.E. Decreau, private communication, 1979). The most important is the one due to the imperfect estimation of the coupling impedance between the plasma and the spheres of the antennas. But it is also difficult to evaluate the effects of the photocloud and of the thermal sheet around the satellite.

Now the true data, on which we base the estimation of the WDF, are the auto and cross-spectra of the field components. They are estimated from finite Fourier transforms of the signal. The practical method we use is based on time averaging over short modified periodograms [Welch, 1967]. Let $\varepsilon_{i}(1)$ and $\varepsilon_{j}(1), \quad(1=0$, .... $N-1)$ be the samples of the components $i$ and $j$, taken in the time interval $T$, in the frame of reference of the satellite. The records are sectioned in $\mathrm{K}$ segments, possibly overlapping, of length $L(1=0, \ldots L-1)$. We consider a Parzen window $W(1)$ and, for each segment, form the sequences $\left[\varepsilon_{i}(1) W(1)\right]_{k}$, $\left[\varepsilon_{j}(1) W(1)\right]_{k}$. We then take the finite Fourier transforms of these sequences.

$$
\begin{aligned}
& A_{k}(n)=\frac{1}{L} \sum_{1=0}^{L-1}\left[E_{i}(1) W(1)\right]_{k} \exp -2 k i l n / L \\
& B_{k}(n)=\frac{1}{L} \sum_{1=0}^{L-1}\left[E_{j}(1) W(1)\right]_{k} \exp -2 k i 1 n / L
\end{aligned}
$$

and obtain the $\mathrm{K}$ modified periodograms

$$
I_{k}\left(u_{n}\right)=\frac{L}{U}\left\{A_{k}(n) B_{k}^{*}(n)\right\}
$$

where $\omega_{n}=2 \pi n / L$ and

$$
\mathrm{U}=\frac{1}{\mathrm{~L}} \sum_{1=0}^{\mathrm{L}-1} \mathrm{~W}^{2}(1) .
$$

The spectral estimate is the average of these periodograms

$$
S_{i j}\left(\omega_{n}\right)=\frac{1}{K} \sum_{k=1}^{K} I_{k}\left(\omega_{n}\right) ;
$$

it has a complex value for $i \neq j$ and a real value for $i=j$. Such a procedure is well-suited for a multi-component analysis as regards the computer memory which is needed.

We assume that this estimate is unbiased $\left(\left\langle s_{i j}\right\rangle=s_{i j}\right)$ and that the variance of the mean is equal to the variance of the $I_{k}$ divided by the number $\mathrm{K}\left(\operatorname{var}\left\{\operatorname{Re}\left[\mathrm{s}_{\mathrm{i}}\right]\right\}=\mathrm{K}^{-1} \operatorname{var}\left\{\operatorname{Re}\left[\mathrm{I}_{\mathrm{k}}\right]\right\}\right.$; $\left.\operatorname{var}\left\{\operatorname{Im}\left[\mathbf{s}_{i j}\right]\right\}=\mathrm{K}^{-1} \operatorname{var}\left\{\operatorname{Im}\left[\mathrm{I}_{\mathrm{k}}\right]\right\}\right)$. The first assumption tends to be true when the spectra are stationary in frequency, the second when the number $K$ is large enough and when the $I_{k}$ have a Gaussian distribution. The two assumptions are more easily fulfilled (which does not mean they are completely fulfilled) for phenomena of the hiss type rather than of the chorus type. Note that the variances calculated are of the same order as the ones estimated from the relations: var $\left\{R_{e}\left[S_{i j}\right]\right\}=S_{i j} S_{j j} / \sqrt{B T}$ where $B$ is the filter $b$ andwidth and $T$ is the observation time.

Some operations are required as we attempt to reconstruct from these estimates the spectra of the wave field components. We must correct, as accurately as possible, for the rotation of the satellite (the spin period is of the order of $10 \mathrm{rpm})$ and then for all the distortions intro- 
duced by the electronic and onboard treatment.

To minimize the effect of the rotation of the satellite, we consider segments containing a very low number of samples, typically $L=16,32$, or 64. Between the first and the last samples the rotation is negligible (from 0.6 to 2 degrees). The resolution in frequency is still reasonable, since it is respectively equal to $46.5,23.25$ or $16.625 \mathrm{~Hz}$.

The following operations are made in this order. The periodograms $I_{k}\left(\omega_{n}\right)$ are individually computed in the frame of reference of their first point. At each frequency $\omega_{n}$, the sampling errors are corrected by multiplying $I_{k}\left(\omega_{n}\right)$ by the factor $\exp \left[-i \omega_{n} \tau_{i j}\right]$, where $\tau_{i}$ is the time shift between the samples of the components $i$ and $j$. Then the transfer function characterizing the electronic distortions is applied. The periodograms are recalculated in the frame of reference of the first periodogram (correction for rotation) and averaged. Finally the spectral matrix, so obtained, is placed in the Cartesian coordinate system of section 2. The values of the power spectral densities at the original frequency $\omega_{0}$ are equal to the values of the power spectral densities at the frequency $\omega_{n}$, the two frequencies being such that $\omega_{0}=\omega_{S F A}+\omega_{n}-300 \mathrm{~Hz}$ with $\omega_{S F A}$ the centra1 frequency of the SFA which is considered.

For the sake of simplicity we consider that the $\hat{\mathrm{s}}_{i j}$ 's so obtained are unbiased, and that the variances of their real and imaginary parts are the variances, in the appropriate reference sys- tem, of the estimates defined in (23). This is obviously an optimistic approximation. For a better evaluation we should take into account the errors in our corrections.

As already pointed out there are more uncertainties in the electric data than in the magnetic ones. A way of testing the validity of the electric measurements is to compare the values of the refractive index obtained on the one hand from the plasma parameters measurements and, on the other hand, from the ratio $\mathrm{E} / \mathrm{B}$, assuming that we have whistler mode propagation along $\vec{B}_{0}$ [Scarf et al., 1969]. In the first case the refractive index, denoted $n_{p}$, is written

$$
\mathrm{n}_{\mathrm{p}}^{2}=\frac{\Pi_{\mathrm{e}}^{2} / \Omega_{\mathrm{e}}^{2}}{\omega / \Omega_{\mathrm{e}}\left(1-\omega / \Omega_{\mathrm{e}}\right)}
$$

$\Omega_{e}$ is deduced from the magnetometer data (S331) and $\Pi_{e}$ from the wave active experiments ( $\$ 301$ and $\$ 304$ ) or by examination of the resonances of the natural waves. In the second case, the refractive index, noted $n_{W}$, can be written ( $T$. Neubert, private communication, 1979)

$$
\mathrm{n}_{\mathrm{w}}^{2}=\mathrm{c}^{2} \mathrm{~B}^{2} / \mathrm{E}_{\perp}^{2}
$$

where $E_{\perp}$ is the component of $\vec{E}$ perpendicular to the $\overrightarrow{\mathrm{K}}$ vector. An estimate of $\mathrm{B}^{2}$ is obtained from the magnetic autospectra $\left(c^{2} B^{2}=c^{2} \mu^{2} H^{2}\right.$ $\left.=\widehat{\mathrm{S}}_{44}+\widehat{\mathrm{S}}_{55}+\widehat{\mathrm{S}}_{66}\right)$, and an estimate of $E_{1}^{2}{ }_{\text {from }}$ the electric autospectra $\left(E_{1}^{2}=\hat{\mathrm{S}}_{11}+\hat{\mathrm{S}}_{22}\right)$. For

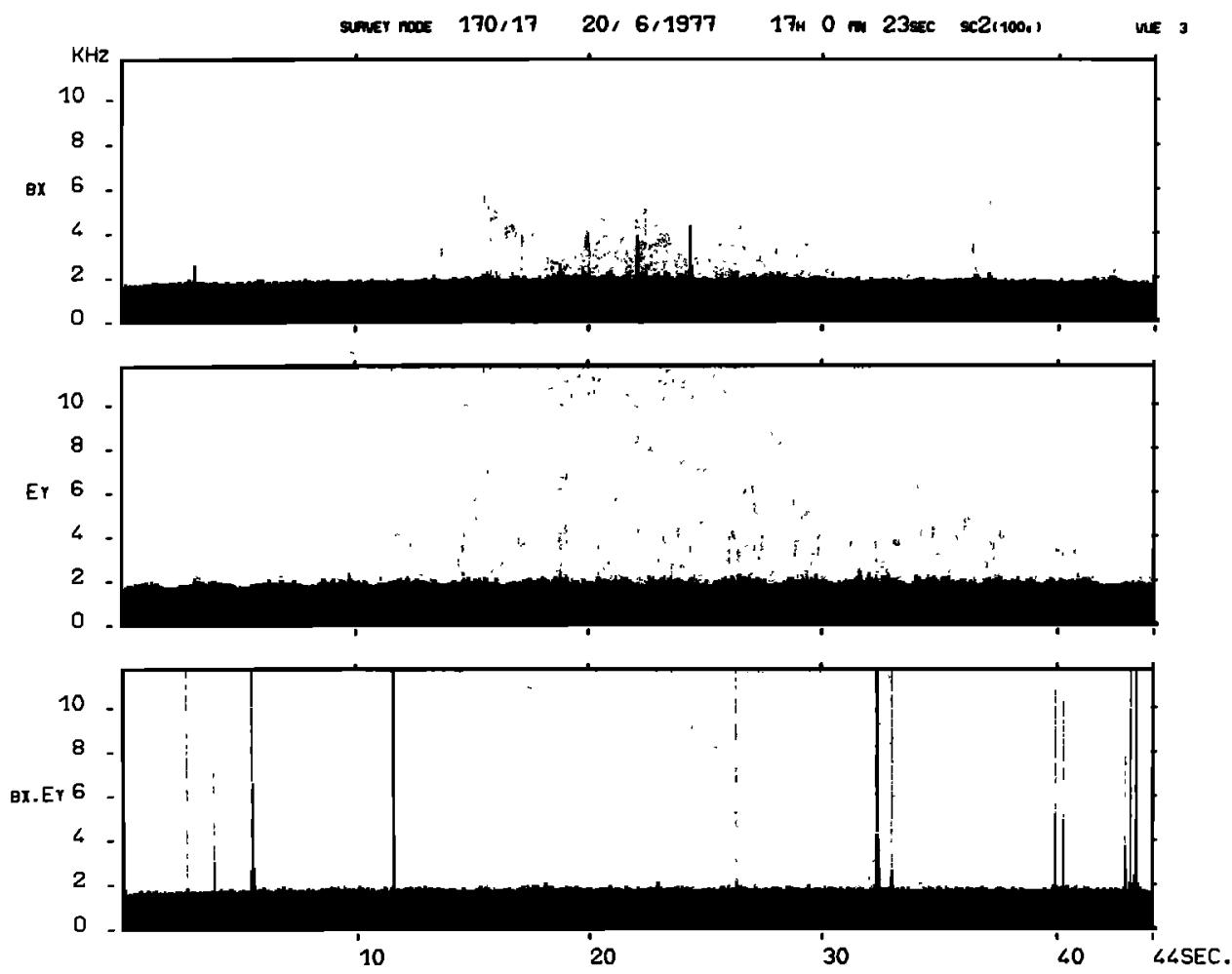

Fig. 3. Auto and cross-power spectra on $\mathrm{b}_{\mathrm{x}}$ and $\mathrm{E}_{\mathrm{y}}$ for the VLF emission event on June 20, 1977, around 1700 UT. The orbit parameters are Local Time LT = 1842; distance in earth radii, Dist $=5.48 ; \mathrm{L}=$ value, $\mathrm{L}=6.18$; geomagnetic Latitude, $\mathrm{LAT}=19^{\circ} 67^{\prime}$; geographic latitude and longitude, latitude $=19^{\circ} 51^{\prime}$, longitude $=17^{\circ} 41^{\prime}$; angle between the $\vec{B}_{0}$ direction and the spin of the satellite, $\alpha=7^{\circ} 13^{\prime}$. The electron gyrofrequency estimated from the onboard magnetometer (experiment S331) is $\Omega_{e}=5.94 \mathrm{kHz}$. The plasma frequency estimated from the active experiments $\mathrm{s} 301$ and $\mathrm{s} 304$ is $\Pi_{e}=60.18 \mathrm{kHz}$. 
oblique propagation $n_{p}$ and $n_{w}$ are respectively low and highe estimates of $n$.

On 20 cases analyzed up to now, using only the small electric antennas for the electric measurements, and at frequencies between $500 \mathrm{~Hz}$ and $3 \mathrm{kHz}$, we have found values of $n_{p}$ and $n_{w}$ such that $0.5 \leqslant n_{p} / n_{w} \leqslant 1.3$, which indicates relatively good consistency between the electric and magnetic measurements. The agreement seems to be still better when the long radial booms are used for estimating $E_{y}$ ( $T$. Neubert, private communication, 1979).

Other tests could be applied to assess the validity of the electric component. According to the linearity of equations (2-a), the equalities that exist between the $a_{i j}$ 's [Storey and Lefeuvre, 1980] ought to hold for the $\hat{s}_{i j}$ 's at least in the limit of the errors in the data. We could expect to have $\operatorname{Re}\left(\hat{\mathrm{S}}_{14}\right) \simeq-\operatorname{Re}\left(\hat{\mathrm{S}}_{25}\right)$; $\operatorname{Im}\left(\hat{S}_{15}\right) \simeq \operatorname{Im}\left(\hat{S}_{24}\right)$ and $\operatorname{Re}\left(\hat{S}_{36}\right) \simeq 0$. Unfortunate1y these equalities are very rarely verified simultaneously, which limits our expectations for the electric data. Furthermore, in the applications we emphasize the results obtained from the magnetic data.

\section{Applications to Geos 1 Data}

Magnetic components. We begin with a detailed study of a VLF emission that nearly fulfills a11 the conditions required by the two methods of analysis. Later we shall deal with more difficult cases. As a first step, the analysis is performed with the magnetic data only. The electric data, when available, will be added later.

The first example chosen is a hiss event recorded June 20, 1977, around 1700 UT. Its auto and cross-spectrograms on $b_{x}$ and $E_{y}$ are represented in Figure 3 . They have been obtained from the onboard correlator measurements [Jones, 1979]. A minor portion of the emission is available at the output of the 3 magnetic SFAs which have a $300-\mathrm{Hz}$ bandwidth and sweep the frequency range $0-10 \mathrm{kHz}$ in steps of $0.69-\mathrm{s}$ duration. The maximum averaged power spectra has been obtained after 1700.44 when the SFAs are centered around $600 \mathrm{~Hz}$. We have performed our analysis at $530 \mathrm{~Hz}$ (for all the sections the resolution frequency is $47 \mathrm{~Hz}$ ). The phenomenon is fairly stationary in time and frequency. The ratio $\omega / \Omega_{e}$ is very small ( 0.09$)$ and one can expect stable solutions.

Contours of the dirichlet kernels and maximum entropy solutions are represented in Figure 4 for $M=7,8,9$. The solutions obtained for $M \leqslant 6$ appear to be of little value and have not been plotted. Effectively, the first kernels vary roughly in $\phi$ as $\sin 2 \phi$ and $\cos 2 \phi$, and we cannot readily distinguish between solutions at $\phi_{o}$ and at $\phi_{0}+\pi$.

The dirichlet kernels solutions cannot be accepted as such, in view of the extent of the zones where there are negative values. However, they provide us with useful information. They can effectively be considered as filtered representations of each possible solution. Although the transfer function $\mathrm{A}\left(\cos \theta, \phi ; \cos \theta_{0}, \phi_{0}\right)$ of the filter presents important side lobes (see Figure 2 for $\theta_{0}=30^{\circ}$ and $\phi_{0}=30^{\circ}$ ), we are sure to determine at least the maximum of the WDF. We cannot be so certain about the interpretation of the secondary peaks, which might be positive side lobes of the main peak. However, in the present case, because of the amplitudes displayed, we can expect that there are two maxima of the WDF.

As $M$ varies from 7 to 9 , the data $\hat{P}_{k}$ are progressively better fitted. As forecast, the prediction parameter reaches the value zero for $M=9$. But at the same time, the instability increases, and $Q$ goes from 0.28 to 0.61 . If the solutions for $M=7$ and 8 are quite similar, the one obtained for $M=9$ is very different. The peaks have a tendency to appear close to the resonance angle, which generally happens in the case of instability. The phenomenon is more noticeable when $\omega / \Omega_{e} \geqslant 0.3$. In these cases, $Q$ takes the values of the order of 2 and more, and the solution at $M=9$ bears no resemblence at all to the ones obtained at $M=7$ and $M=8$. Finally the solution we select for the hiss event of June 20,1977 , is the one obtained for $M=8$ (Figure $4 \mathrm{~b}$ ). It is stable and fits the data well. Its resolving power is superior to the solution for $M=7$.

The maximum entropy solutions (Figure $4 \mathrm{~d}, 4 \mathrm{e}$, 4f) are clearly more satisfactory, since they obey the positivity constraint. However, they exhibit the same general features as the dirichlet kernels solutions, which is not surprising considering that the latter can be regarded as filtered representations of any solution and therefore of the maximum entropy solution. A noticeable discrepancy would mean that the solution obtained from the maximum entropy method is not a satisfactory solution to our inverse problem, which is theoretically possible since it has never been demonstrated that a maximum entropy solution always exists. Thus, we can validate the maximum entropy solution by comparing it with the dirichlet kernels solution. This enables us to interpret the prediction parameter $P_{r}$ with much more freedom. A value of $\mathrm{P}_{r}$ reasonably larger than 1 does not necessarily mean that the maximum entropy solution is invalid. In fact, it generally indicates that there is an inaccurate estimate of the errors on the data.

As for the dirichlet kernels solutions, going from $M=7$ to $M=8$ we improve the resolving power without changing the main features. At $i=9$ the instability appears to gather all of the energy in the neighborhood of the resonance angle. It is still relatively weak $(Q=1.6)$ in comparison with the instability observed in the cases where $\omega / \Omega_{e} \geqslant 0.3$ ( $Q$ can reach values up to $10^{3}$ ) and for which the initial solutions are completely destroyed. The solution to be selected here is the one obtained at $M=8$. Its prediction parameter still has a relatively high value

$\left(\mathrm{P}_{\mathrm{r}}=2.56\right)$ but, as was already mentioned, this is not surprising, in view of inaccuracy in the estimation of errors in the data. We could try to improve the fit in taking $\varepsilon^{\prime}<M$ in the inequality (20). However, we must come to grips with numerical instabilities in the integration of $\left(2^{\prime}\right)$.

Finally, the WDF associated with the hiss event of June 20,1977, is a two-peaked function. The main peak is centered around $\theta=60^{\circ}$ (or $\left.120^{\circ}\right)$ and $\phi=50^{\circ}$ (or $\left.230^{\circ}\right)$, and the secondary one around $\theta=25^{\circ}$ (or $155^{\circ}$ ) and $\phi=220^{\circ}$ (or $40^{\circ}$ ). We shall see later that the electric data 
enables us to completely remove the ambiguity between the waves propagating in the $\overrightarrow{\mathrm{B}}_{\mathrm{o}}$ direction and in the opposite direction. We note that the two peaks are separated by $\simeq 180^{\circ}$ in $\phi$, and that the wave normal directions are approximately concentrated in an off-meridian plane $\left(\phi \simeq 50^{\circ}\right)$. In the hypothesis of propagation in the $\vec{B}_{0}$ direction, the major part of the energy is due to waves whose wave normals make an angle of $\sim+60^{\circ}$ wi th $\vec{B}_{o}$, but a non-negligible part is due to waves whose wave normals make an angle of $\sim-25^{\circ}$ with $\vec{B}_{0}$. Although the plane wave hypothesis-is not valid here, the wave normal direction given by Mean's method is consistent with these results, since it gives as values of $\theta$ and $\phi$ : $\theta=24^{\circ} 5, \phi=48^{\circ}$. This wave normal direction is in the off-meridian plane defined above. Its angle with $\vec{B}_{0}$ is halfway between $+60^{\circ}$ and $-25^{\circ}$. Note that considering only the Mean's solution, one would have concluded that the waves propagate with wave normal directions almost parallel to the earth's magnetic field direction, which is not our conclusion after a WDF analysis.

To study the stationarity of the WDF in time and in frequericy, we have chosen to work on another hiss event, slightly more difficult to deal with but having more distinctive, analyzable steps of the SFAs. The record was made on July 14, 1977, around 1900 UT. The auto and crossspectrograms for $b_{x}$ and $E_{y}$ are represented in Figure 5. They are spin-modulated. We consider three different steps of $0.69 \mathrm{~s}$. The first at 1900.43 , and the third at 1900.50 correspond to step number 4 of the SFAs. In both cases the analysis is made at $1.17 \mathrm{kHz}$, which makes $\omega / \Omega_{e}=0.12$. The second step is taken at 1901.43, about $300 \mathrm{~Hz}$ above. The analysis is made at $1.46 \mathrm{kHz}$ with a ratio $\omega / \Omega_{\mathrm{e}}=0.16$. Only the maximum entropy solutions are presented (Figure 6). They have been checked against dirichlet kernels solutions. For $1.17 \mathrm{kHz}$, the solutions at $M=8$ have been found to be stable enough to be selected. For $1.46 \mathrm{kHz}$, perhaps because of the increase of the ratio $\omega / \Omega_{e}$, the solution at $M=8$ is slightly unstable $\left(Q=1.30, P_{r}=1.47\right)$ and we have preferred the one derived at $M=7$. In any case, they are very similar. The modulation of the signals, observed in the frame of reference of the satellite, increases the inaccuracy in the estimates of the $\hat{s}_{i j}$ 's. This explains the values reached by the prediction parameter $\left(P_{r}=4.11\right.$ in the first step).

Despite the difference in time and in frequen$c y$, the three WDF exhibit roughly the same characteristics. They present a strong peak, around $\theta=70^{\circ}$ (or $110^{\circ}$ ) and $\phi=230^{\circ}$ (or $50^{\circ}$ ). There is still a certain amount of energy around $\theta=55^{\circ}\left(\right.$ or $\left.125^{\circ}\right)$ and $\phi=90^{\circ}$ (or $270^{\circ}$ ), but a wel1-detached secondary peak only exists for the first step. The propagation is concentrated around an off-meridian plane. It turns out that the spin modulation seen on the spectrograms is due, on the one hand, to the concentration of energy around the main peak, with the consequences that the $\vec{E}$ and $\vec{H}$ polarization planes do not fluctuate very much, and on the other hand, to the inclination of the plane of rotation of the antennas relative to those polarization planes (the spin axis of the satellite makes an angle $\alpha=46.47^{\circ}$ with $\vec{B}_{0}$ ).

Chorus data are much more difficult to analy- ze in terms of the WDF for two reasons. First, the waves are not stationary in frequency, and if they are stationary in time it is over very short periods; 5 to 8 elementary spectra are used to estimate the data $\hat{\mathbf{P}}_{\mathbf{k}}$ while up to 64 are considered in the hiss case. Secondly, they are recorded in zones where the ratio $\omega / \Omega_{e}$ is high (from 0.3 to 0.6 ). Then we have more uncertainty in the data and more linear dependency of the $\hat{\mathrm{q}}_{\mathrm{k}}$ 's. These are two additional sources of instability in the solutions. Nevertheless, the analysis may turn out to be meaningful because the signal is nearly coherent and the signal/ noise ratio is very high.

To illustrate this we have chosen to present the WDF associated with the strong chorus recorded in the August 14, 1977, data around 1000 UT. Their auto and cross-spectrograms on $b_{x}$ and $E_{y}$ are represented in Figure 7. Each of the main emissions contains several distinctive elements. We have focused on one of them that is part of the emission seen around 1001.05 on the spectrograms. The WDF analysis has been performed for $1.76 \mathrm{kHz}\left(\omega / \Omega_{\mathrm{e}}=0.4\right)$. The maximum entropy solutions at $M=6,7$ and 8 are about the same and are correctly related to the corresponding dirichlet kernels solutions. At $M=9$ the WDF splinters, which is a good indication that the instability occurs between $M=8$ and 9 , and not before. We have only given here the maximum entropy solution derived for $M=7$ (Figure 8). Its stability parameter is still high $(Q=1.63)$, but for the reasons discussed above we believe that this is due to a bad estimation of the errors on the data. The WDF is a two-peaked function. The waves propagate with their wave normals in an off-meridian plane $\left(\phi \simeq 105^{\circ}\right)$. Under the hypothesis of propagation in the $\vec{B}_{0}$ direction, the energy is almost equally shared between longitudinal waves $\left(\theta \simeq 25^{\circ}\right)$ and resonant waves $\left(\theta \simeq+59^{\circ}\right)$.

One might wonder why all the WDF presented here are two-peaked functions. Is there a bias in the methods used? We believe that this is not so for the following two reasons. First, a onepeaked function has already been associated with a chorus event recorded onboard Ogo 5 [Lefeuvre and Delannoy, 1979]. Second, as we shall see in the second part of this section, one-peaked functions are found for artificial signals emitcted from the ground and observed on Geos. We conclude that these two-peaked functions which we have generally derived are real. They correspond to a common feature of the natural emissions observed in the equatorial region on the Geos sate1lite. We shall discuss this point in more detail in section 5 .

Magnetic and Electric Components. We have calibrated our method on artificial signals, emitted from the ground transmitter Omega located in Norway, and received onboard Geos [Ungstrup et al., 1978]. Such signals are a priori of the plane wave type and are supposed to propagate vertically. Furthermore, they have already been analyzed in terms of wave normal direction by $T$. Neubert (private communication, 1979). The only point is that at the time they were recorded on Geos (second half of December 1977) the magnetometer data was in error; it is therefore proposed to derive the $\vec{B}_{0}$ direction from a model field line. Following Ungstrup 
$\theta$

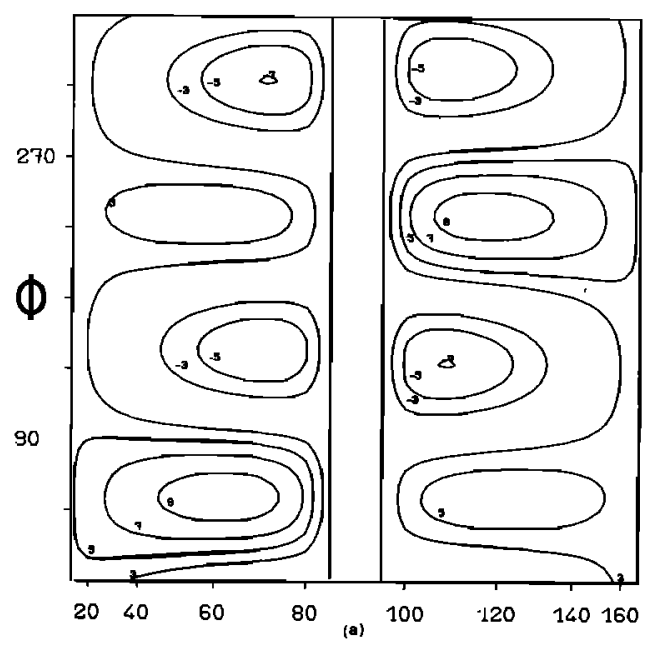

$\Theta$

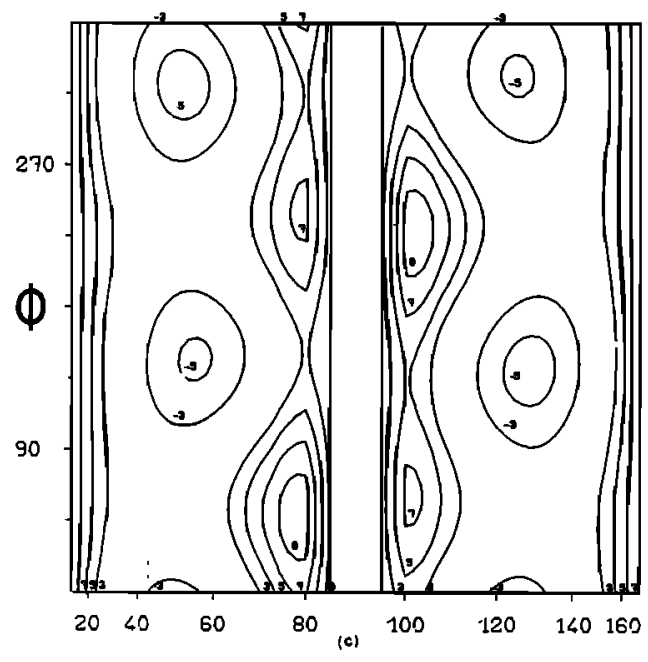

$\Theta$

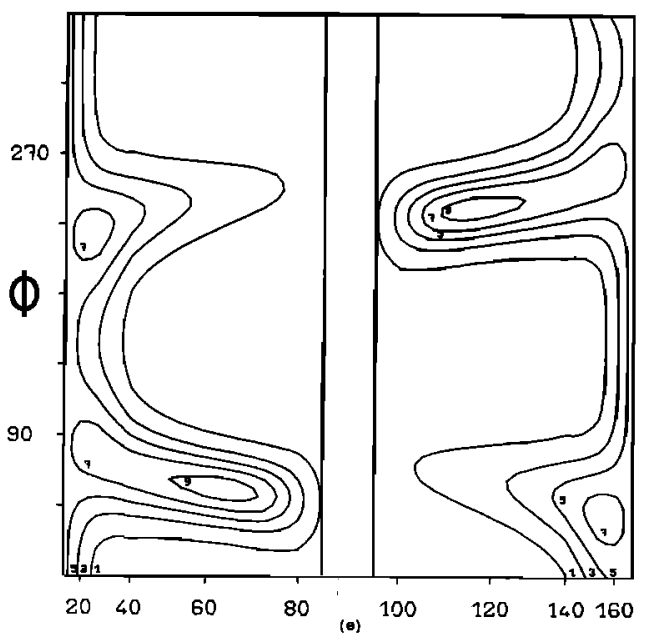

$\Theta$

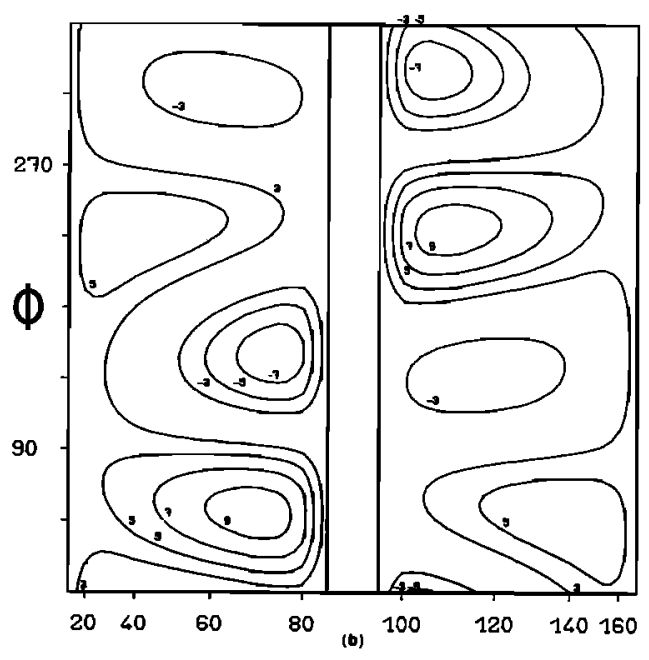

$\theta$

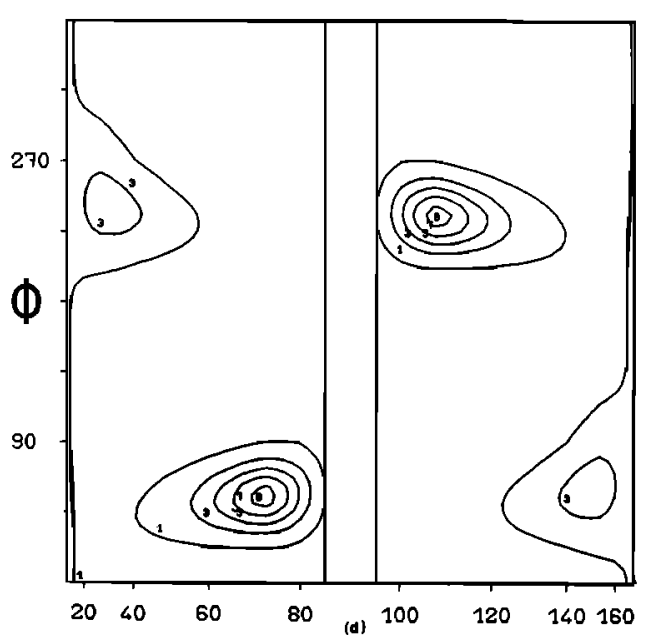

$\Theta$

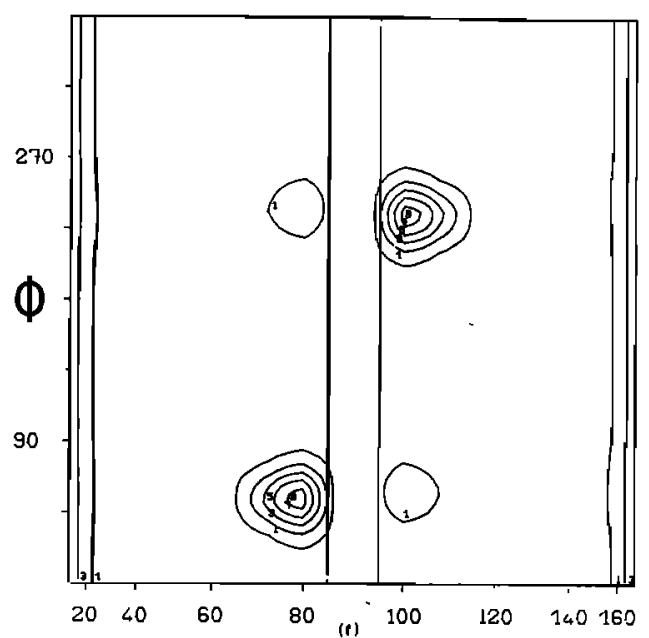

Fig. 4. Contours of dirichlet kernels and maximum entropy solutions for $0.69 \mathrm{~s}$ of the VLF emission on June 20, 1977, starting around 1700.44 (Figure 3). The analysis is made at the frequency $\omega_{0}=0.530 \mathrm{kHz}$ where the signal is maximum $\left(\sim 3.210^{-5} \mathrm{\gamma}^{2} / \mathrm{Hz}\right)$. The characteristics of the plots are the same as those in Figure 2. $Q$ and $P_{r}$ are the stability and prediction parameter (see text). On the left the dirichlet kernels solutions are represented; (a) $N=9, M=7, Q=0.28, P_{r}=0.47$. (b) $N=9, M=8$, $\mathrm{Q}=0.35, \mathrm{P}_{\mathrm{r}}=0.27$. (c) $\mathrm{N}=9, \mathrm{M}=9, \mathrm{Q}=0.61, \mathrm{P}_{\mathrm{r}}=0$. On the right the maximum entropy solutions are represented: (d) $\mathrm{N}=9, \mathrm{M}=7, \mathrm{Q}=0.39, \mathrm{P}_{\mathrm{r}}=2.81$. (e) $\mathrm{N}=9$, $M=8, Q=0.76, P_{r}=2.56$. (f) $N=9, M=9, Q=1.60, P_{r}=2.21$. 

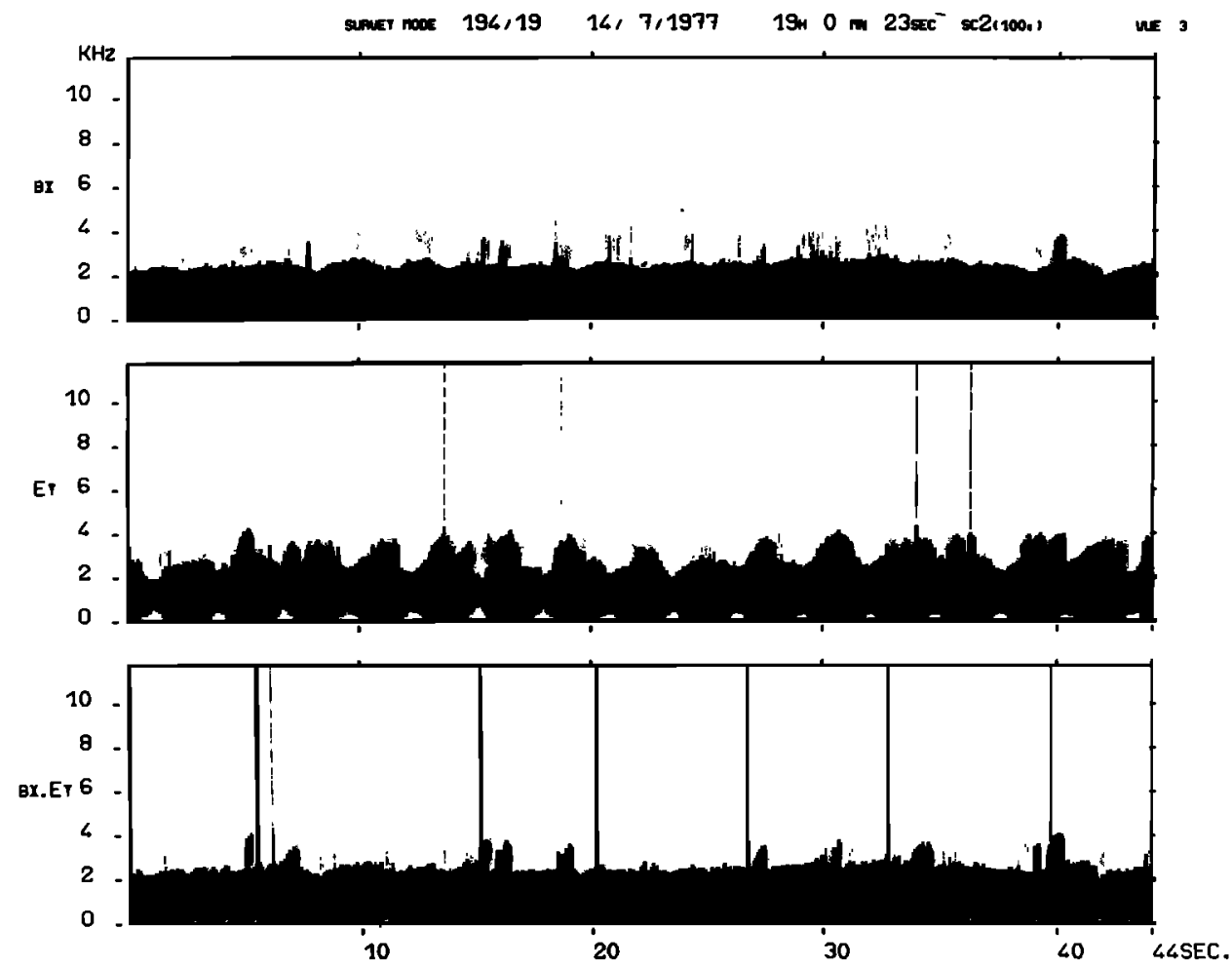

Fig. 5. Auto and cross-power spectra on $b_{x}$ and $E_{y}$ for the VLF emission event on July 14, 1977, around 1900 UT. The orbit parameters are LT $=1885$, Dist $=3.88, \mathrm{~L}=5.31$, LAT $=31^{\circ} 2^{\prime}$, lat $=26^{\circ} 07^{\prime}$, Long $=-9^{\circ} 36^{\prime}, \alpha=46^{\circ} 47^{\prime}$. The estimated plasma parameters are $\Omega_{e}=9.32 \mathrm{kHz}, \Pi_{e}=58.35 \mathrm{kHz}$.

et al. [1978] we have used Kosik's model calculations [Kosik, 1978].

About 1 second of the Omega signal recorded on December 27, 1977, around 0800.54 UT has been analyzed. Its frequency is above $10 \mathrm{kHz}$, so no display of its spectra is available. Its averaged power spectra shows a single fine line at $10.2 \mathrm{kHz}$. The solution obtained from the magnetic components only has been represented in Figure 9a. It is quite consistent with the results given by the Mean's method $\left(\theta=54^{\circ}\right.$ or $126^{\circ}, \phi=196^{\circ}$ or $16^{\circ}$ ) and by the T. Neubert cross product method (values of $\theta$ and $\phi$ for the latter are not given here, since the calculation is made for each sample of the signal, which on1y allows a qualitative comparison). As was previously explained, the WDF so derived is a onepeaked function. When adding the information relative to the long $\mathrm{E}_{\mathrm{y}}$ antenna, we obtain solutions such as the one of Figure $9 \mathrm{~b}$; the ambiguity about the direction of the wave normals has been completely removed. The Omega signal propagates with wave normal directions contained in a narrow cone centered on $\theta=126^{\circ}, \phi=16^{\circ}$. The earth's magnetic field being oriented downwards in the northern hemisphere, the wave propagates vertically as assumed.

What about the analysis of the magnetic and electric data for the natural emissions? The situation is in fact quite variable. We have cases for which it seems practically impossible to derive a stable solution that correctly fits all the data, while in some other cases we are apparently successful. What is to be singled out, besides the restrictions already imposed on the electric measurements (section 3)? There are at least four important factors. First, we are more successful when using the $y$ component of the electric field measured by the long radial electric antenna rather than the small axial electric antenna; in such a case, as for the Omega signal, we even prefer not to make use of the other two components. Second, when using the electric components measured only by the small electric antennas, we believe that there is more chance to derive a stable solution when the plasma is dense and so when the small electric antennas are outside the Debye sphere, the threshold is obviously not easy to fix. Third, we must try to avoid electric data that is contaminated by pulses due to the shadow effect of the longest antennas on the smallest ones. Fourth, we have more linearly independent items of information when the ratios $\omega / \Omega_{e}$ and $\Omega_{e} / \Pi_{e}$ are small and more errors on the data when the phenomenon is poorly stationary in time and in frequency.

Having all these constraints in mind, we review the natural events discussed in the first part of this section and for which only the small antennas were connected. The hiss event recorded on October 26,1977 , almost fulfills the conditions. Its maximum entropy solution is presented in Figure 10. The ambiguity about the wave direction is completely removed; the two waves propagate in the $\vec{B}_{0}$ direction. We attribute the displacement of the peaks, relative to Figure $4 \mathrm{f}$, to the inaccuracy in the electric data. The number of information items effectively 
$\Theta$

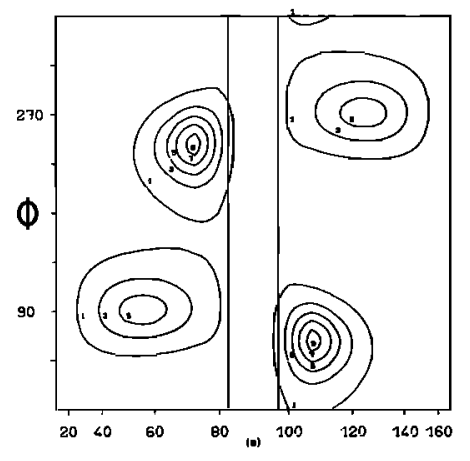

$\theta$

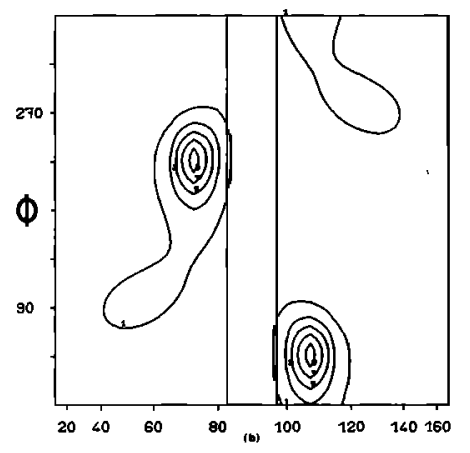

$\theta$

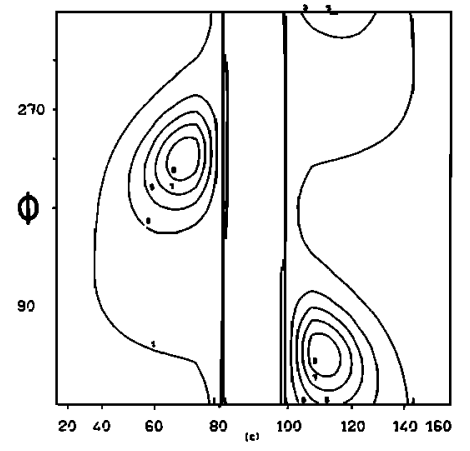

Fig. 6. Contours of maximum entropy solutions for three different $0.69-s$ intervals of the VLF event on July 14, 1977 (Figure 5). (a) Around 1900.43 and at $\omega_{0}=1.17 \mathrm{kHz} ; \mathrm{N}=9, \mathrm{M}=8, Q=$ $0.67, P_{r}=4.11$. (b) Around 1901.5 and at $\omega_{0}=$ $1.17 \mathrm{kHz} ; \mathrm{N}=9, \mathrm{M}=8, \mathrm{Q}=0.66, \mathrm{P}_{\mathrm{r}}=3.06 ;$ (c) Around 1901.4 and at $\omega_{0}=1.46 \mathrm{kHz}, Q=0.61$, $P_{r}=1.61$.

used might seem very low ( $M=9$ for $N=36)$, but as soon as we increase it an instability occurs. In fact, comparing Figures $4 \mathrm{f}$ and 10 , one sees that the electric data have only been used to remove the ambiguity about the direction. They do not seem to be accurate enough to improve the determination of the solution derived from 8 magnetic sources of information, i.e., to refine the peaks or even find new ones. In any case, the reader will note that in the two analyses the items of information are not equivalent.
There is a difference between using 9 magnetic inputs and selecting, among the 36 electric and magnetic items of information, the 9 least $1 i-$ nearly dependent ones. In the first case (Figure 4f) we obtain an ambiguous and unstable solution. In the second (Figure 10) the solution is unambiguous and stable. This demonstrates the importance of ranking the items of information as illustrated in Figure 2.

On July 14, 1977, we do not obtain convergence towards a satisfactory stable solution. The WDF derived for the chorus event of August 14, 1977, is more stable but it is not completely satisfactory. The two peaks are strongly displaced relative to the ones pointed out in Figure 8 and it is obvious that the magnetic and electric components are not coherent between themselves. However, it seems clear that the wave packets propagate in the $\vec{B}_{0}$ direction.

Natural emissions have also been recorded when the long electric antenna $E_{y}$ was connected. An example is given in Figure 11. It is a kind of modulated hiss similar to the activity on July 14, 1977, with the difference that the spin axis of the satellite is nearly perpendicular to $\overrightarrow{\mathrm{B}}_{\mathrm{O}}$. The analysis was made at $1.07 \mathrm{kHz}$, around 1056.30. Here the duration of the step is $2.75 \mathrm{~s}$ which is more convenient for a good estimation of the data $\hat{\mathrm{P}}_{\mathrm{k}}$. Two solutions are given (Figure 12). The first one has been obtained from the 7 most linearly independent magnetic data, the second from the 10 most $1 i-$ nearly independent magnetic and $E_{y}$ data. We clearly see two wave packets, located approximately in the same off-meridian plane $\left(\phi \sim 80^{\circ}\right)$, propagating in the negative $\overrightarrow{\mathrm{B}}_{\mathrm{o}}$ direction. The energy centered around $\theta=-45^{\circ}$ is almost negligible relative to that centered around $\theta \simeq 58^{\circ}$. $A$ third packet is also present in the $\vec{B}_{0}$ direction. However, we believe that it is nothing but the residual 'image' of the main one centered at $\theta=120^{\circ}$. We completely destroy the solution if we increase the $M$ value keeping $N=16$ or if we try other $M$ values while considering the entire set of electric and magnetic data $(\mathrm{N}=36)$.

This last example does not give a WDF as well defined as in the cases of the Omega signal and the hiss event of June 20, 1977. But it better illustrates what we can generally expect from the Geos data.

\section{Summary and Discussion}

The two methods of estimation of the wave distribution function examined in this paper are complementary. The maximum entropy solutions, smooth and positive everywhere, might appear sufficient but they are particular solutions. The dirichlet kernels solutions are very poor if we consider that they do not obey the positivity constraint. However, they give an idea about the main features of a11 the possible solutions. As a result we have found that there is not a large discrepancy between the maximum entropy solutions and the infinity of other possible solutions. This strongly increases confidence in the maximum entropy solutions.

In determining the WDF it is important to realize that the items of information about the wave 


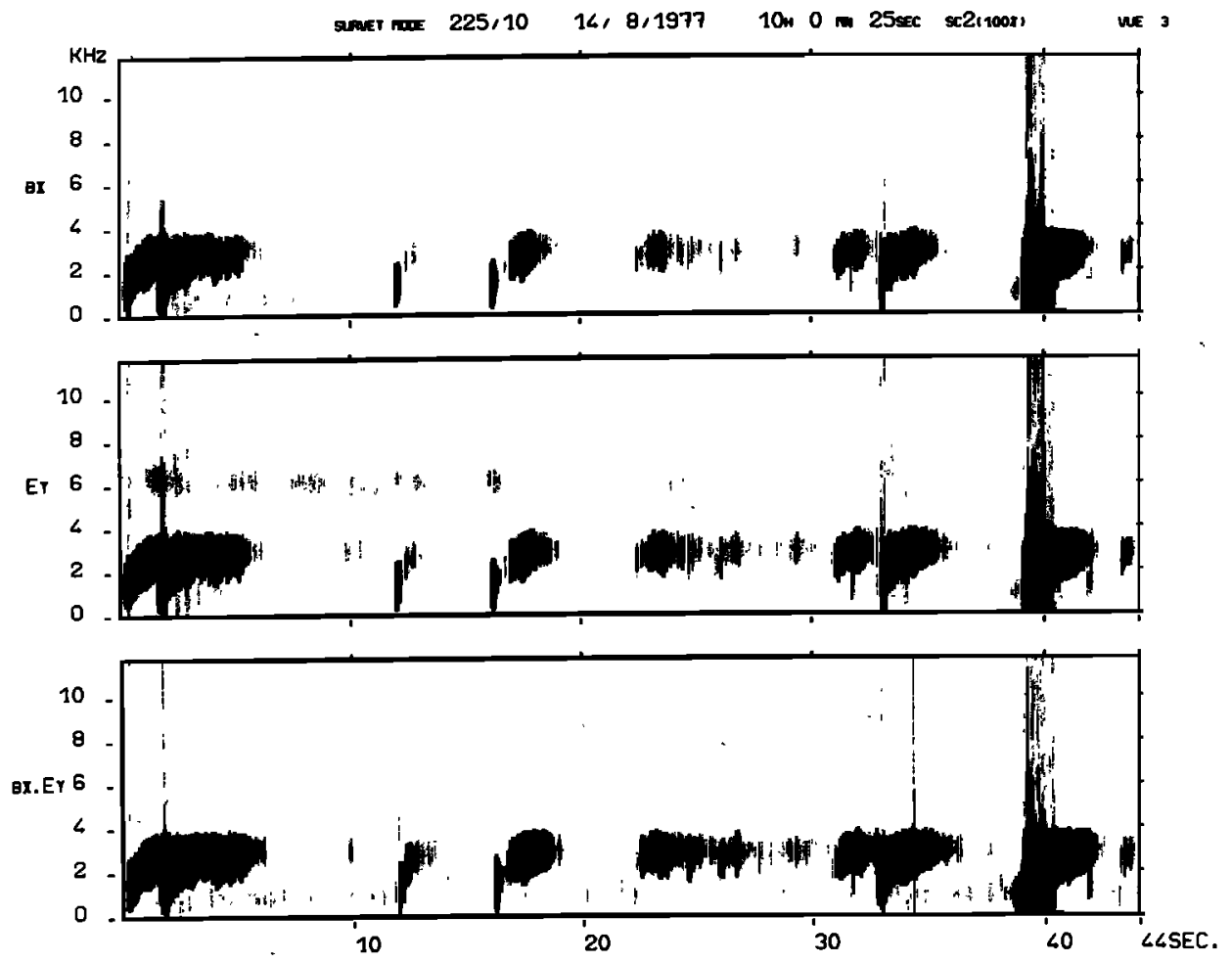

Fig. 7. Auto and cross-spectra on $b_{x}$ and $E_{y}$ for the VLF emission event on August 14, 1977, around 1000 UT. The orbit parameters are: LT $=1148$, Dist $=5.95, \mathrm{~L}=6.01$, LAT $=5^{\circ} 7^{\prime}$, 1at $=0^{\circ} 80^{\prime}$, long $=25^{\circ} 10^{\prime}, \alpha=12^{\circ} 06^{\prime}$. The estimated plasma parameters are $\Omega_{e}=4.42 \mathrm{kHz}, \Pi_{e}=23.7 \mathrm{kHz}$.

fields do not all have the same significance. From 9 poorly chosen items of information we obtain an unstable and ambiguous solution that is physically meaningless (Figure 4f). From 9 other carefully selected items we have a stable and unambiguous solution that we can physically interpret (Figure 10). Therefore, the ranking of the items of information relative to their linear frequency is fundamental. On this matter it must be noted that the truncation scheme is applied to the transformed, and not the real, data. Since each of the transformed data is a linear combination of all the original data, removing transformed data does not mean removal of any of the original data. It is therefore useless to try to design an ideal experiment based on restricting the number of sources of data. The ideal experiment remains a 6-component measurement from which we extract the most relevant information. In practice, as we have found, an accurate measurement of one electric component can be more informative than less reliable measurements of all three electric components. The extent of processing errors in the data must also be taken into account.

If we assume a perfect measurement of al1 3 magnetic and all 3 electric components, there are basically two conditions for application of the methods. First, the phenomenon under study must be stationary in time and frequency. Second, the local plasma parameters must be such that $\omega / \Omega_{e}$ and $\Omega_{e} / \Pi_{e}$ tends toward zero. Hopefully, analysis can be performed when those two condi- tions are not completely fulfilled, but the results may be less realiable.

Practically, we have seen that if we accept an ambiguity in sign of the direction of propagation with respect to the earth's magnetic field, we can almost always determine a WDF from

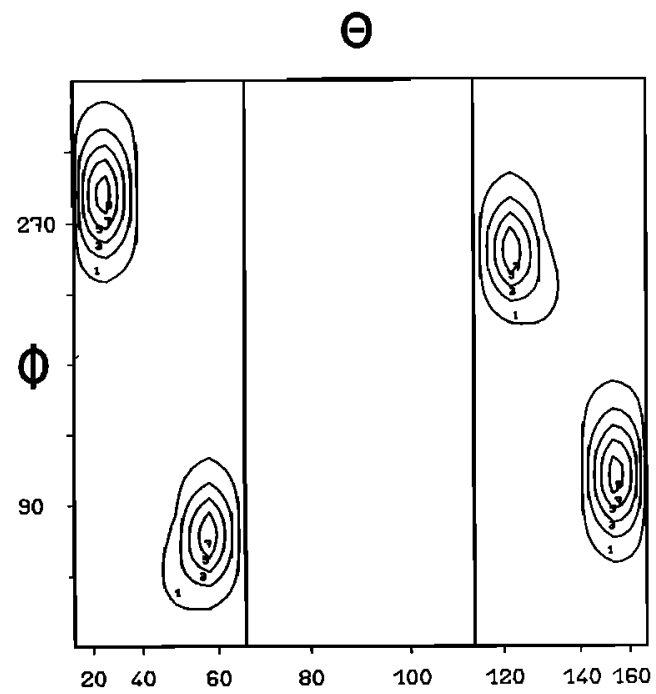

Fig. 8. Contours of the maximum entropy solution for $0.07 \mathrm{~s}$ of the VLF emission of Figure 7 . The analysis is made at the frequency $\omega_{0}=1.76 \mathrm{kHz}$ were the signal is maximum $\left(5.110^{-4} \gamma^{2} / \mathrm{Hz}\right)$. We have $\mathrm{N}=9, \mathrm{M}=7, \mathrm{Q}=1.63, \mathrm{P}_{\mathrm{r}}=1.90$. 
$\Theta$

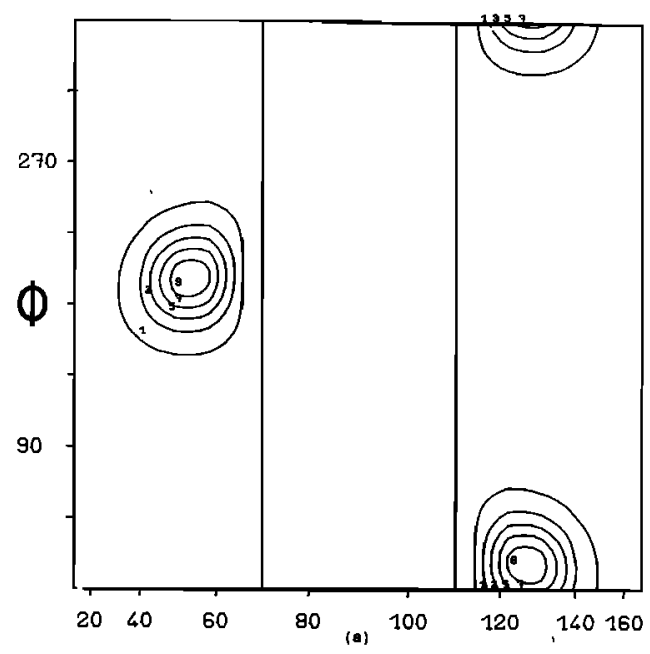

$\Theta$

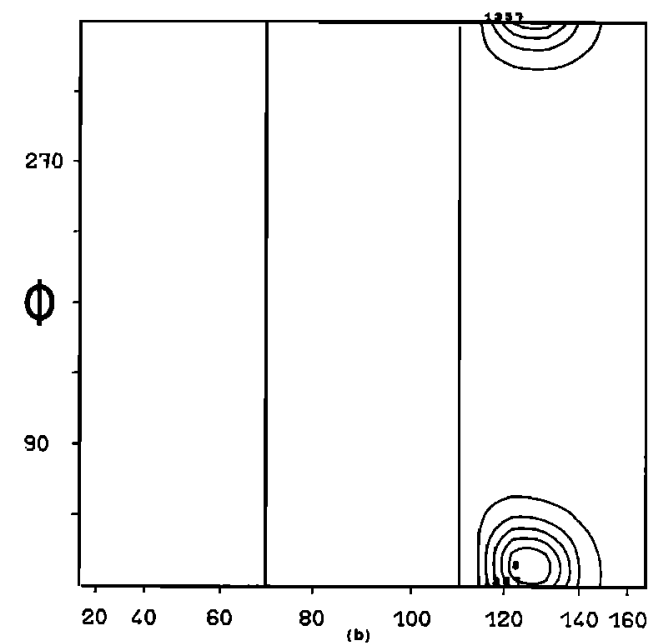

Fig. 9. Contours of maximum entropy solutions for $1 \mathrm{~s}$ of the Omega signal received onboard Geos on December 27, 1977, around 0808.54. The wave frequency is $\omega_{0}=10.2$ $\mathrm{kHz}$. The electron gyrofrequency is estimated from a mode $1, \Omega_{e}=30 \mathrm{kHz}$, and the plasma frequency is extrapolated from previous onboard measurements, $\Pi_{e}=100 \mathrm{kHz}$. The orbit parameters are LT $=1020$, Dist $=3.50, \mathrm{~L} \simeq 4$, lat $\simeq 20^{\circ}$, long $\simeq 20^{\circ}$. We have (a) $N=9, M=7, Q=0.49, P_{r}=5.37$. (b) $N=16, M=10, Q=0.32, P_{r}=$ 7.59 .

the measurements of the three magnetic components only. The Geos 1 electric measurements of ten permit the removal of the ambiguity, but are not accurate enough to improve determination of the solution, i.e., to refine the peaks or eventually find new ones.

Omega data, for which the plane wave hypothesis is generally valid, and to which the classical methods of analysis are therefore applicable, have enabled us to calibrate our own methods. We have checked that waves emitted from the ground and observed onboard Geos 1 during a pass of the satellite above the emitter have their wave normals oriented vertically and concentra-

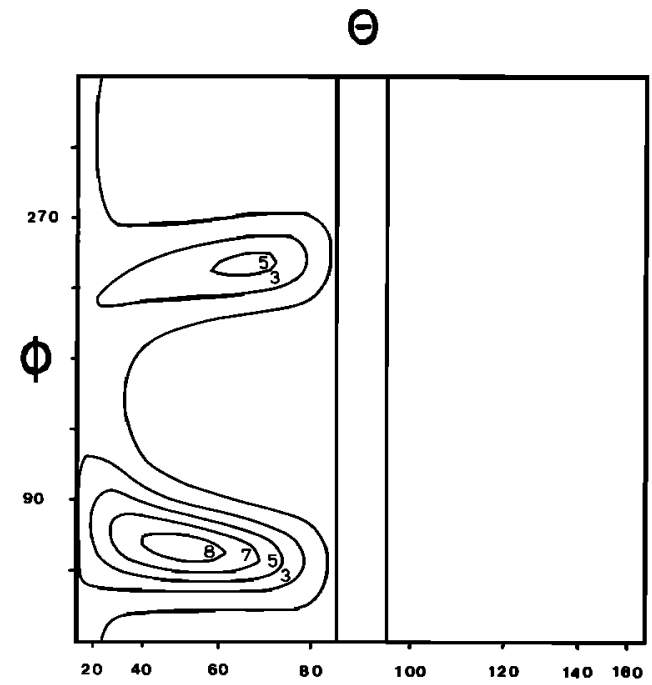

Fig. 10. Maximum entropy solution for the hiss event on June 20, 1977 (see Figures 3 and 4), for $\mathrm{N}=36$ and $\mathrm{M}=9$. The quality parameters are $\mathrm{Q}=0.43, \mathrm{P}_{\mathrm{r}}=2.33$. ted in a narrow cone whose axis is practically coincident with the unique wave normal derived from the classical methods.

Some new results have been obtained about natural VLF emissions. The most striking feature we have found is that for hiss as well as for chorus the wave energy is of ten contained within two different wave packets whose wave normals are concentrated in the same off-meridian plane and which are oriented in the same manner relative to the earth's magnetic field direction $\vec{B}_{0}$. This is summed up in Figure 13. The wave normals $\vec{K}_{1}$ and $\vec{K}_{2}$ characterize the peaks of the WDF. It has been found difficult to give an idea of the extension of each wave packet, the wave normals being unevenly spread around $\overrightarrow{\mathrm{K}}_{1}$ and $\vec{K}_{2}$. The direction of the waves for July 14, 1977, was not known; we have assumed that they were propagating with their longitudinal components in the $\overrightarrow{\mathrm{B}}_{\mathrm{o}}$ direction. No such assumption was needed for the three other cases, since the ambiguity was removed in the course of the analysis. $\overrightarrow{\mathrm{K}}_{1}$ has been systematically taken to indicate the more energetic wave packet. The wave energy is equally shared between the two wave packets in what we suppose to be the generation region (geomagnetic latitude close to zero). The wave packet associated with $\overrightarrow{\mathrm{K}}_{2}$ tends to fade out outside of this generation zone.

Comparisons with previous analyses are difficult; having assumed the presence of a plane wave, the authors obviously found one single wave normal direction. In the case of a two-peaked function, this single wave normal direction indicates an average direction, differing in $\theta$ and $\phi$ from the directions of either peak (see the analysis of the June 20, 1977, event). The only study which seems to be relevant is the one by Burton and Holzer [1974] on the chorus data observed onboard the 0go 5 satellite at 


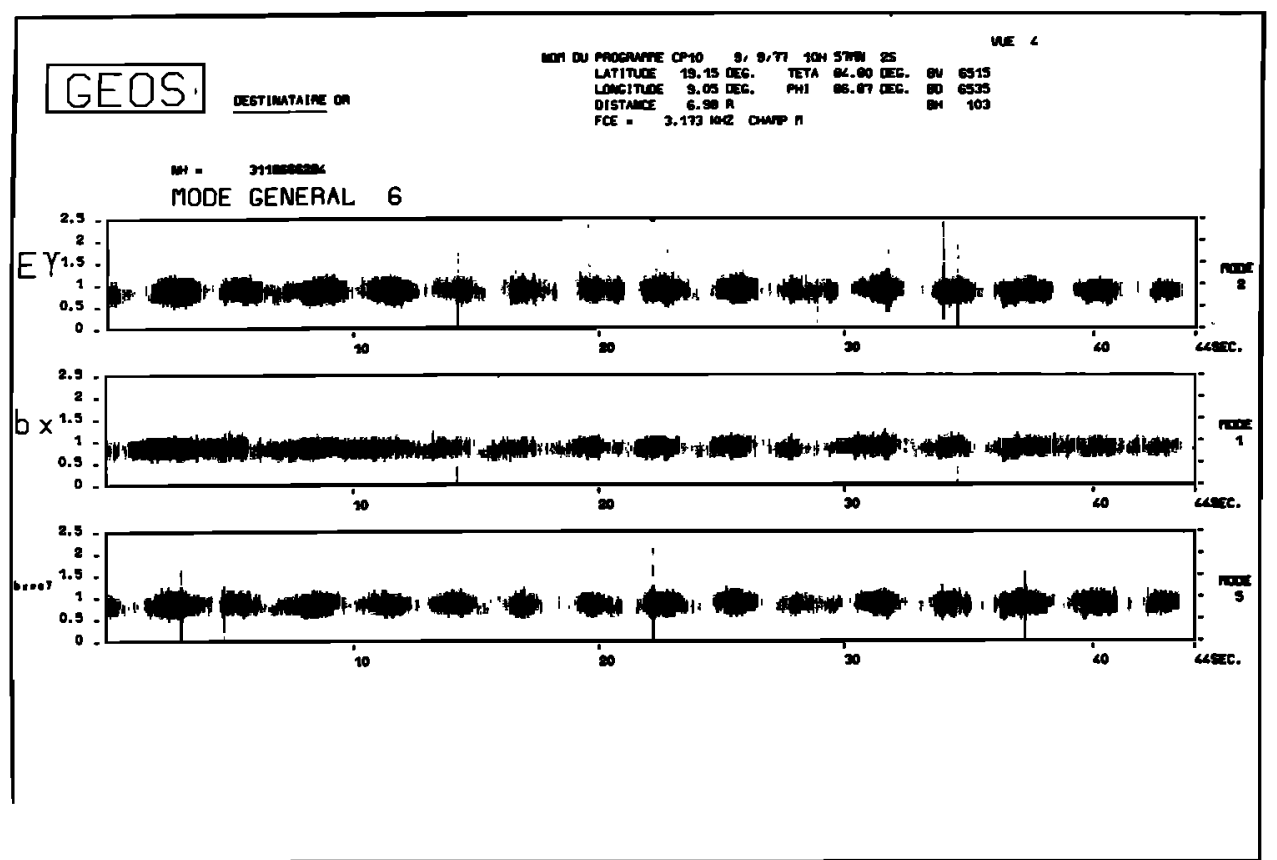

Fig. 11. Auto and cross-spectra on $b_{x}$ and $E_{y}$ for the VLF emission on September 9, 1977, around $1056 \mathrm{UT}$. The orbit parameters are $\mathrm{LT}=1178$, Dist $=4.47, \mathrm{~L}=7.98$, LAT $=20^{\circ} 7^{\prime}$, lat $=19^{\circ} 02^{\prime}$, long $=9^{\circ} 40^{\prime}, \alpha=94^{\circ} 74$. The estimated plasma parameters are $\Omega_{e}=3.17 \mathrm{kHz}, \Pi_{e}=17.24 \mathrm{kHz}$.

$5.9 \leqslant L \leqslant 7.9$. They used the plane wave approximation over short time intervals $(0.01 \mathrm{~s})$ but after collecting a sufficient number of wave normal directions, they built a statistical wave normal distribution. This enabled them to find two-peaked functions similar to ours in what they called the generation zone. The two wave packets propagate in the same hemisphere relative to $\vec{B}_{0}$; their wave normals are concentrated in the same plane. However, we disagree on three points: first, Burton and Holzer found that the two nlane waves were in the geomagnetic meridian plane;

$\theta$

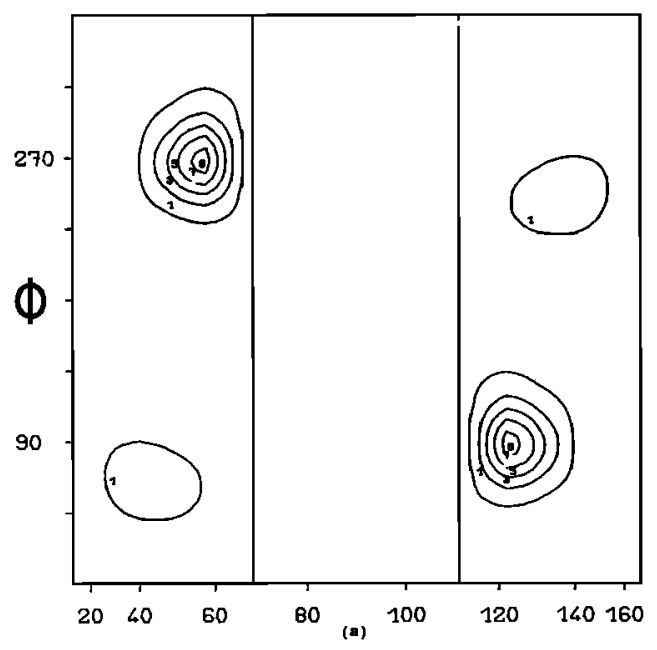

second, the dispersion in $\theta$ is admittedly very sma11; third, the waves normals are said to be evently distributed between positive and negative $\theta$. Now, although we do not refer to the same set of data, it turns out that we find different results because we use different methods. The methods are only comparable if the observed field is of a single plane wave over the time interval of $0.01 \mathrm{~s}$ that Burton and Holzer need to estimate each couple $(\theta, \phi)$. If not, we believe their $\theta$ and $\phi$ values are biased, including their averaged values.

$\Theta$

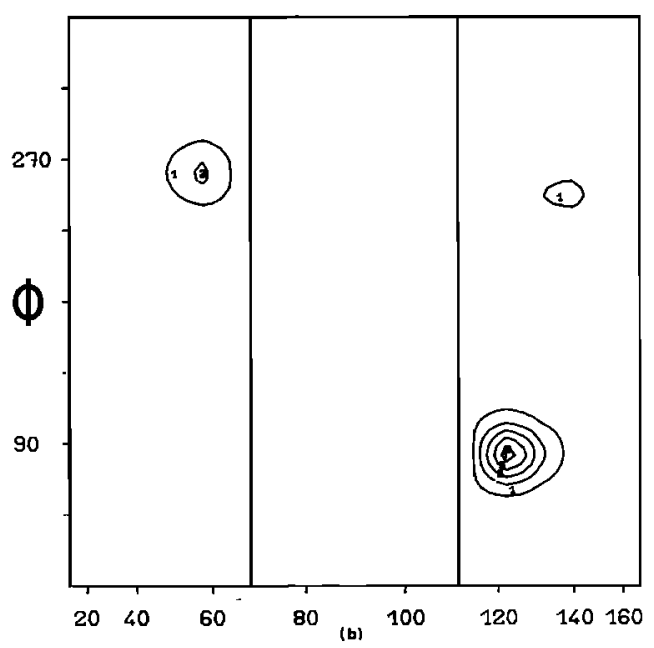

Fig. 12. Maximum entropy solution for $0.69 \mathrm{~s}$ of the VLF event on September 9, 1977 , starting around 1056.30 (Figure 10). The analysis was made at $\omega_{0}=1.07 \mathrm{kHz}$ where the signal is maximum $\left(3.10^{-5} \gamma^{2} / \mathrm{Hz}\right)$. (a) $N=9, M=7, Q=0.64, P_{r}=2.34$. (b) $\mathrm{N}=16, \mathrm{M}=10, \mathrm{Q}=0.87, \mathrm{P}_{\mathrm{r}}=4.80$. 


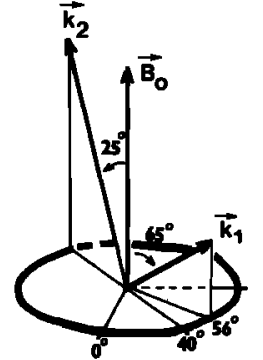

(a)

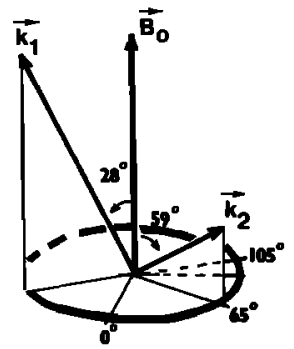

(c)

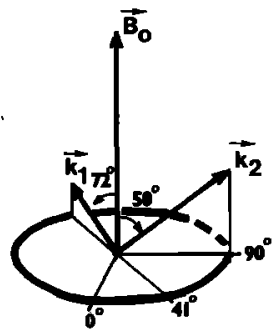

(b)

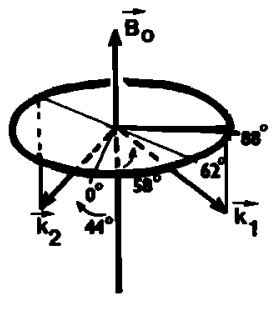

(d)
Fig. 13. Averaged wave normal directions. $\overrightarrow{\mathrm{K}}_{1}$ has been systematically taken to indicate the more energetic direction. (a) June 20, 1977. (b) July 14, 1977. (c) August 14, 1977, (d) September 9, 1977.

In fact, in the equatorial region it is not surprising to find wave normals in off-meridian planes. Thorne [1969] showed that it is only far from this region that wave normals are collimated into meridian planes. However, the simultaneous observation of sma11 and large $\theta$ angles, separated by $\sim 180^{\circ}$ in $\phi$, is much more unexpected. It tends to support the idea of waves propagating from a single source, but we have to find a way to generate and propagate these waves to the satellite.

One tentative explanation could be as follows. Let us assume an isotropic source, i.e., a source exciting waves in all allowable wave normal directions (this hypothesis is not necessarily supported by current theories of wave-particle interactions). At frequencies below $\Omega_{\mathrm{e}} / 2$ there is a central cone of radiation about $\vec{B}_{0}$ within which rays with three different wave normal angles may a11 propagate in the same direction [Helliwe11, 1965]. In particular, rays in the $\vec{B}_{0}$ direction result from wave normal angles of zero and $\pm \theta g$, where $\theta g$ is the Gendrin [1961] angle at which $d(n \cos \theta) / d \theta=0$. We denote as $\theta_{i}$ the point of inflection on the refractive index surface, and following Burtis [1974] identify the waves with local wave normal $|\theta|<\theta_{i}$ as the 'convex' mode and those with $|\theta|>\theta_{\mathbf{i}}$ as the 'convave' mode. At a given distance from the source, the satellite only 'sees' the parts of the 'convex' and 'concave' modes which have been conveyed by rays which join the source to the satellite. At short distances from the source, the medium is nearly homogeneous and one can expect to observe two wave normal directions associated with a single ray and such that $\left|\theta_{1}\right|<\theta_{i}$ and $\left|\theta_{2}\right|>\theta_{i}$. The satellite not being in general on the same line of force as the sourçe, the ray direction is not exactly parallel to $\vec{B}_{0}$, and the only waves of the concave mode which can be observed are the ones opposite to the convex mode at $\theta_{1}$, i.e., in our original notation, the ones distant by $\pi$ in $\phi$.

At larger distances, the inhomogeneity must be taken into account. Burtis [1974] calculates that there exists a large region of space in which each point can be reached by waves emitted from the same source, in different wave normal directions, and that have followed different ray paths. In that case, the local wave normals differ significantly from the wave normals at the point of emission. It is difficult to identify the concave and convex modes, but we can expect that the $\phi$ values have not been drastically modified so that $\phi_{2}-\phi_{1} \sim \pi$.

The chorus event of August 14, 1977, could represent the case of a measurement close to the sourçe. The ray directions calculated from $\vec{K}_{1}$ and $\vec{K}_{2}$ create angles of $\beta=-2^{\circ}$ and $-13^{\circ}$ with $\vec{B}_{0}$, but an estimation, using a two-plane wave mode 1 [Buchalet and Lefeuvre, this issue] gives about the same angle for the two ray directions. The other events discussed in this paper are definitely observed far from the source. The ray directions associated with the couple $\vec{K}_{1}$ and $\vec{K}_{2}$ are opposite (for instance $\beta_{1}=11^{\circ}$ and $\beta_{2}=-10^{\circ} 5$ for June 20,1977 ) as can be expected from the proposed model.

Other explanations are possible. Using ray tracing to analyze chorus observed onboard Scatha, Koons [1979] suggests that, at frequencies below $\Omega_{e} / 2$, a part of each chorus element propagates with small $\theta$ angles while the other part propagates with large $\theta$ angles. This is not in contradiction with our findings but appeals to a different model of wave generation.

Acknowledgments. The authors are most gratèful to L. R. O. Storey who initiated the work on the determination of the wave distribution function. They are also indebted to T. Neubert (Danish Space Research Institute), who provided them with preliminary results from the Omega data, and to their colleague L. J. Buchalet for helpful discussions. Their thanks to $B$. De La Porte (C.R.P.E., Issy les Moulineaux), D. Barria, G. Charles, C. Huc, and G. H. Mingot (Centre $\mathrm{Na}^{-}$ tional d'Etudes Spatiales, Toulouse) for their competent and constant assistance in the data handing. Their appreciation is extended to their colleague L. Cairo and the members of the Centre Interuniversitaire de Calcul de la Region Centre for assistance in obtaining the final displays. The final manuscript was written and prepared by $F$. Lefeuvre during his visit to the Radioscience Laboratory, Stanford University, thanks to a NSF-CNRS fellowship. Comments received at the Radioscience Laboratory, particularly from $D$. L. Carpenter, were very useful in interpreting the results. Thanks are also due to $G$. Daniels who greatly improved the English of the paper, and to, K. Dean and C. Marcadet for preparation of the final typescript.

The Editor thanks E. J. Smith and C. C. Harvey for their assistance in evaluating this paper. 


\section{References}

Arthur, C. W., R. L. McPherron, and J. D. Means, A comparative study of three techniques for using the spectral matrix in wave analysis, Radio Sci., 11, 833, 1976.

Backus, G., and F. Gilbert, The resolving power of gross earth data, Geophys. J. R. Astron. Soc., 16, 169, 1968.

Backus, G., and F. Gilbert, Uniqueness in the inversion of inaccurate gross earth data, Phil. Trans. R. Soc. London, 266, 123, 1970.

Buchalet, L. J., and F. Lefeuvre, One- and twodirection models for VLF electromagnetic waves observed onboard Geos 1, J. Geophys. Res., this issue.

Burtis, W. J., Magnetospheric chorus, Tech. Rep. 3469-3, Radioscience Lab., Stanford Electron. Lab., Stanford Univ., Stanford, Calif., 1974.

Burton, R. K., and R. E. Holzer, The origin and propagation of chorus in the outer magnetosphere, J. Geophys. Res., 79, 1014, 1974.

Cornilleau-Wehrlin, N., J. Etcheto, and R. K. Burton, Detailed analysis of magnetospheric ELF chorus: Preliminary results, J. Atmos. Terr. Phys., 30, 1201, 1976.

Gendrin, R., Le guidage des whistlers par le chamị magnétique, Planet. Space Sci., $\underline{5}$, 274,1961 .

Gilbert, F., Ranking and winnowing gross earth data for inversion and resolution, Geophys. J. R. Astron. Soc., 23, 125, 1971.

Grard, R., Interprétation de mesures de champs électromagnétiques TBF dans la magnétosphère, Ann. Geophys., 24, 995, 1968.

Helliwe11, R. A., Whistlers and Related Ionospheric Phenomena, pP. 40-43, Stanford University Press, Stanford, Calif., 1965.

Jackson, D. D., Interpretation of inaccurate, insufficient and inconsistent data, Geophys. J. R. Astron. Soc., 28, 97, 1972.

Jones, D., Auto- and cross-correlation analysis techniques onboard spacecraft, Ann. Telecommun., 34, 187, 1979.

Kodera, $\bar{K}$., R. Gendrin, and $C$. de Villedary, Complex representation of a polarized signal and application of the analysis of ULF waves, J. Geophys. Res., 82, 1245, 1977.

Koons, H. C., Generation of chorus emissions in the outer magnetosphere, paper presented at AGU Fall Meeting, San Francisco, Calif., December 1979.

Kosik, J. C., The uses of past and present magnetospheric field models for mapping fluxes and calculating conjugate points, Space Sci. Rev., 22, 161, 1978.

Lefeuvre, F., Analyse de champ d'ondes électromagnétiques aléatoires observés dans la magnétosphère à partir de la mesure simultanée de leurs six composantes, thèse de doctorat d'état, University of Orléans, Orléans, France, 1977.

Lefeuvre, F., and C. Delannoy, Analysis of a random electromagnetic wave field by a maximum entropy method, Ann. Telecommun., 34, 204, 1979.

Lefeuvre, F., and L. R. O. Storey, The analysis of a 6-component measurement of a random electromagnetic wave field in a magnetoplasma, 2 ,
Mode1 identification, Tech. Note CRPE/41, Centre de Recherches en Physique de 1'Environnement, Orléans, France, 1977.

Loisier, G., Cornilleau-Wehrlin, N., and R. Gendrin, Normale d'onde de signaux variant dans le Temps, Ann. Teleconmun., 34, 214, 1979.

McPherron, R. L., C. T. Russe11, and P. J. Coleman, Fluctuating magnetic field in the magnetosphere, 2, ULF waves, Space Sci. Rev., $13,411,1972$.

Means, J. D., The use of the three-dimensional covariance matrix in analyzing the properties of plane waves, J. Geophys. Res., 77, 5551, 1972 .

s-300 Experimenters, Measurements of electric and magnetic wave fields and of cold plasma parameters onboard Geos 1, Preliminary results, Planet. Space Sci., 27, 317, 1979.

Sabatier, P. C., Remarks on approximate methods in geophysical inverse problems, Proc. R. Soc. London, 1, 49, 1974.

Sabatier, $\bar{P}$. C., On geophysical inverse problem and constraints, J. Geophys., 43, 115, 1977.

Scarf, F. L., C. F. Kennel, R. W. Fredericks, I. M. Green, and G. M. Crook, AC fields and wave-particle interactions, in Particles and Fields in the Magnetosphere, edited by B. M. McCormac D. Reide1, Hingham, Mass., 1969.

Shawhan, S. D., The use of multiple receivers to measure the wave characteristics of very-1owfrequency noise in space, Space Sci. Rev., 10, $689,1970$.

Storey, L. R. 0., Electric field experiments: Alternating fields in the ESRO Geostationary Magnetospheric Satellite, Rep. SP-60, pp. 267279, European Space Research Organization, Neuilly sur Seine, France, 1971.

Storey, L. R. 0., and F. Lefeuvre, Theory for the interpretation of measurements of a random electromagnetic wave field in space, Space Res., $15,381,1974$.

Storey, L. R. 0., and F. Lefeuvre, Analysis of a wave field in a magnetoplasma, 1 , The direct problem, Geophys. J. R. Astron. Soc., 56, 255, 1979.

Storey, L. R. 0., F. Lefeuvre, Analysis of a wave field in a magnetoplasma, 2, The integration kerne 1s, Geophys. J. R. Astron. Soc., 62, 173194,1980 .

Thorne, R.M., Whistler mode propagation off the geomagnetic meridian plane, internal report, Department of Meteorology, University of California, Los Angeles, California, United States, 1969.

Welch, P. D., The use of Fast Fourier transform for the estimation of power spectra: A method based on time averaging over short, modified periodograms, IEEE Trans. Audio Electroacoust., $15,70,1967$.

Wiggins, R. A., The general linear inverse problem: implication of surface waves and free oscillation for earth structure, Rev. Geophys. and Space Physics, 10, 251, 1972.

(Received Apri1 2, 1980; revised September 15, 1980; accepted September 16, 1980.) 